\title{
A new rheological model for thixoelastic materials in subaqueous gravity driven flows
}

\author{
Hermidas Navid ${ }^{1,}{ }^{\text {, }}$, Silva Jacinto Ricardo ${ }^{2}$, Eggenhuisen Joris T. ${ }^{3}$, Luthi Stefan M. ${ }^{1}$
}

${ }^{1}$ Faculty of Civil Engineering and Geosciences, TU Delft, Delft, Netherlands

${ }^{2}$ Marine Geosciences Unit, Ifremer, Brest, France

${ }^{3}$ Department of Earth Sciences, Utrecht University, Utrecht, Netherlands

*Corresponding author : Navid Hermidas, email address : $\underline{\text { n.hermidas@tudelft.nl }}$

\begin{abstract}
:
A new viscoelastic constitutive model for subaqueous clay-rich gravity flows is presented. It is explained that for the materials which exhibit a minimum in their strain controlled flow curves the structure parameter must be a symmetric function of strain rate and stress. Therefore, the destruction of structure within the material is modeled using the dissipation energy. An expression for the elastic strain of the flowing structure is derived. The final set of equations can reproduce the viscosity bifurcation that clay suspensions may exhibit under a given load. This is explained to be important for prediction of the runout distance of clay-rich gravity flows. The ability of the model to reproduce the general response of pasty materials to step stress and step shear rate tests is examined. The model requires four empirical parameters. A methodology is presented for obtaining these parameters and power law functions are given for their calculations for a limited rest time of 3000 seconds. The ability of the model to reproduce the rheological behavior that clay-rich suspensions exhibit under both stress and strain controlled conditions is examined using rheometry tests.
\end{abstract}

\section{Highlights}

- A new rheological model with only four empirical parameters is presented. Our rheological model explains the run-out distances of clay-rich gravity flows. - The structure parameter is a symmetric function of the strain rate and stress. An expression for the elastic strain of the flowing structure is derived. Our rheological model can reproduce the viscosity bifurcation of clay suspensions.

Keywords : gravity flow, thixotropy, clay suspension, viscosity bifurcation 


\section{Introduction}

Clay is present in the majority of deep marine [1], coastal [2, 3], and fluvial environments 3 [4]. For clay-rich grayity flows, presence of clay greatly influences the run-out distance and the 4 internal structure of these flows $[5,6]$.

5 Using their inclined plane experiments, Coussot et al. [7, 8] showed that under a given load, clay 6 suspensions experience viscosity bifurcation, i.e., they either stop flowing altogether or experi7 ence a discontinuous decrease in their viscosity depending on their structural state at the time 8 of the applied stress. They concluded that, for strain controlled measurements, stable flows can occur only when the shear rate is above a critical value. For smaller shear rates the material either fractures or shows shear banding instabilities [9]. These experiments also showed that if a

11 clay-water suspension with a given thickness begins to flow on a certain slope, it will stop only

12 when the thickness is reduced dramatically or when the slope is decreased considerably. This

*Email address for correspondence: N.Hermidas@tudelft.nl

Preprint submitted to Journal of Non-Newtonian Fluid Mechanics

February 28, 2019 
phenomenon is a consequence of the existence of two yield stresses. One which governs the initiation of the flow from rest and is known as the static yield stress, $\tau_{y}$, and the other which governs the abrupt stoppage/freezing of the flow and is known as the dynamic or critical yield stress, $\tau_{c}$. The static yield stress is larger than the critical yield stress and the difference between the two is the factor which governs the run-out distance of clay-water suspensions. The model which will be presented here can capture both the static and the critical yield stresses of clay-water suspensions, and therefore, can be used to accurately simulate the run-out distance of gravity flows.

The most complete anatomy of subaqueous clay-rich gravity flows is composed of three vertically stacked layers [10]. These three regions are characterized in Figure 1(a), which depicts the velocity profile of a clay-rich sediment gravity flow during small scale flume experiments [10].

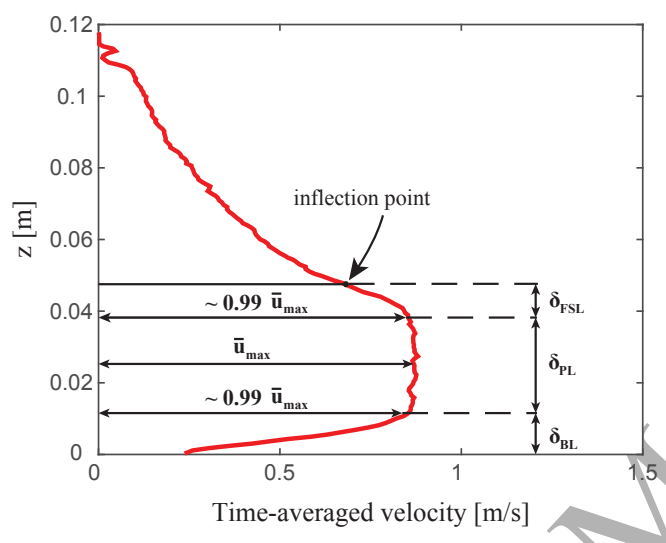

(a) Velocity profile of a $21 \%$ concentration gravity flow

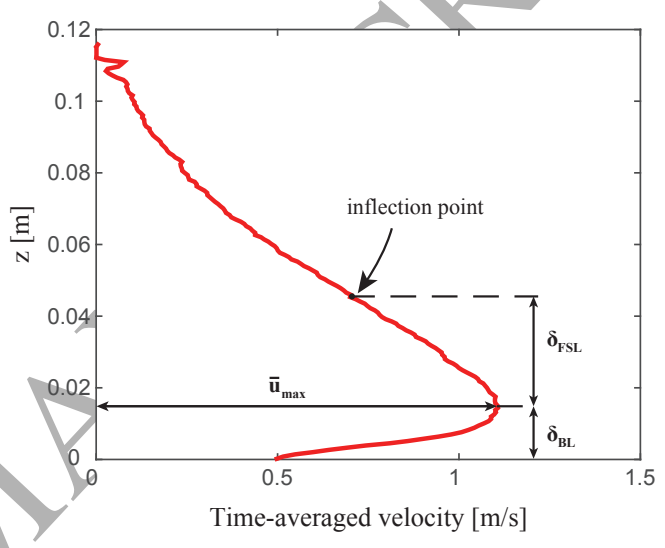

(b) Velocity profile of a $9 \%$ concentration gravity flow

Figure 1: Free shear layer, $\delta_{F S L}$, plug layer, $\delta_{P L}$, and boundary layer, $\delta_{B L}$, regions of sediment gravity flows captured using an Ultrasonic Doppler Velocity probe [10].

The density and velocity gradients that exist on the interface between the sediment flow and the overlying water result in a dilute mixing layer known as the free shear layer. Due to low sediment concentration, the fluid within this layer behaves similar to a Newtonian fluid. The free shear layer is stacked on top of a layer referred to as the plug layer. The behavior of the mixture within this layer resembles that of a gel-like suspension whose yield stress is reached on the interface where the free shear layer and the plug layer meet. The existence and size of the plug layer is dependent on the sediment concentration, and for dilute sediment gravity flows it disappears, as shown in Figure 1(b). Due to the no slip condition between the sediment flow and the bed, the plug layer in the vicinity of the bed is liquefied, producing a third layer known as the boundary layer. The fluid within this layer behaves as a viscoelastic fluid.

Modeling approaches for these flow structures are problematic due to the complex behavior of clay-water suspensions. Clay particles are flat platelets with electrostatically charged surfaces. Once immersed in high concentrations (higher than the 'gelling' concentration) in a medium such as water, they can form two types of structures, namely, the 'card-house flocs' and 'card-pack aggregates' [11]. Presence of these structures are characteristic of many clay-water suspensions which exhibit viscoelastic, yield, shear thinning, and thoxitropic behavior [12].

Thixotropy is generally viewed as the time dependent decrease in the apparent viscosity of a 
fluid under shear due to the break down of the structure [8]. Recovery of a damaged structure ensues once shearing is removed. The concept of simultaneous break down and build up of structure was first discussed by Goodeve [13]. Subsequent models of Moore [14], Hahn et al. [15], and Peter [16] aimed at capturing these simultaneous processes by a rate equation for a scalar structure parameter that is incorporated in the constitutive model. Generalization of these models for the special case of steady, homogeneous, incompressible, irrotational flows, came following the work of Rivlin $[17,18,19,12,20]$. For more general flows, different variations of these models are usually considered where various material characteristics such as yield stress $[21,22]$, elastic modulus, and/or viscosity are considered to be functions of the structure parameter [23, 24, 25, 26, 27, 28]. These functions are generally constructed in such a way as to reproduce the overall behavior of the material.

In contrast to this group of models, which are based on the bulk rheological behavior of the material, a separate branch has evolved over the years which adopts a microstructural approach. The constitutive relations in these models are emergent from the physical considerations regarding the interactions between the material's microstructural constituents $[29,30,31,32]$. While the level of abstraction that these models possess and their added intuition from the microstructural approach is appealing, considerable mathematical manipulation is usually required to express them in terms of macroscopic variables. Therefore, they have not often been employed to describe experimental data sets. The bulk rheological models on the other hand, such as those of Mujumdar et al. [33] or Dullaert and Mewis [34], are written explicitly in terms of the bulk parameters and are therefore easier to employ in engineering applications.

Nonetheless, construction of such bulk models can suffer from ambiguities regarding how various relations should be constructed. One ambiguity has to do with the way the structure model is formulated. For instance, the destruction of structure in such models has been related to various invariants of the deviatoric stress and/or the strain rate tensors depending on the material and the flow field characteristics [27]. Here we will remove this ambiguity by resorting to physical symmetry arguments.

In this study a new rheological model is presented which can be used for modeling clay-rich gravity flows. In order to capture the anatomy of these flows, from the outset a constitutive model is favored that can reproduce the creep behavior of the plug layer, the yielding at the base of the plug, and the visco-elastic behavior within the boundary layer. The Kelvin-Voigt model is here considered very applicable for the elastic solid and the yield regimes, and suitable for the viscous fluid regime. Therefore, similar to the approach taken by Mujumdar et al. [33], the total stress is split into an elastic and a viscous part. However, in contrast to their approach, an expression for the elastic strain of the flowing structure is not assumed. Instead, such an expression is derived based on the structure model and by assuming spring like interactions between clay particles at the micro scale. This reduces the number of empirical parameters required by the model. Generation and destruction of structure is accounted for using a structure model based on the model of Yziquel et al. [27].

We will discuss how the final equations capture the viscosity bifurcation and the yielding and freezing behavior that clay suspensions display under a given load. This is important for accurate prediction of the run-out distance of gravity flows. The most general flow curves which are allowed by the model are discussed. The model response to constant stress and constant shear rate inputs are analyzed [28, 35, 36, 34].

The final constitutive model requires four empirical parameters. A methodology is presented for obtaining these parameters and power law functions are give for their calculation, assuming complete structure recovery within a limited amount of rest time. We will show that the model is 
capable of reproducing the rheological behavior that clay-rich suspensions may exhibit in both stress and strain controlled flow conditions which exist within the free shear and boundary layers of clay-laden environmental flows. This is achieved by comparing the output of the model with stress and strain controlled measurements obtained from rheometry tests.

\section{Rheological model}

\subsection{Connecting micro scale behavior to a macro scale model}

At very short distances two clay particles exert a repulsive force on each other (Born repulsion). At longer distances, this repulsive force is overcome by Van der Waals attraction and therefore two clay particles attract each other. In the absence of other forces, the addition of these two forces results in an equilibrium distance corresponding to the balance of Born repulsion with Van der Waals attraction [37, 38]. A net repulsive force is generated when the particles are brought inside the equilibrium distance, and a net attractive force when the particles are brought outside the equilibrium distance. Therefore, at short distances the interaction of clay particles can be modeled by springs. Although, it is known that at long distances two clay particles may exert a net repulsive force on each other [38], here we assume that this force is weak and the interaction can be modeled as if the spring between the particles is broken.

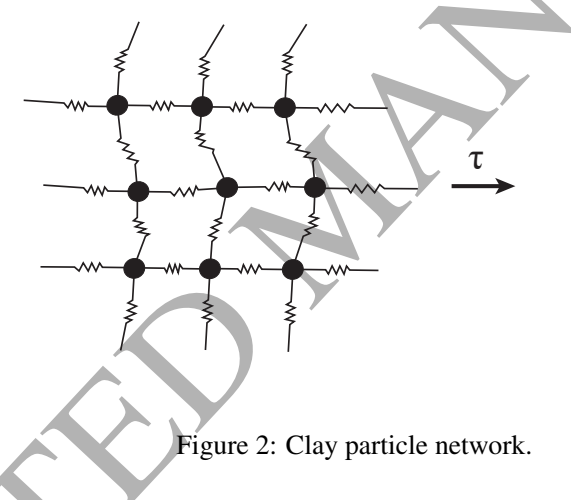

Consider a force applied to a homogeneous and isotropic network of clay particles connected together with springs as in Figure 2. Following the homogeneity and isotropy assumptions, away from the boundaries, the direction in which a force is applied to this network is irrelevant. Furthermore, once equilibrium is reached the internal forces cancel out and the applied force is counteracted by the sum of all the forces that are associated with strains in the direction of the applied force. However, the amount of strain that the whole network experiences in the direction of the applied force is different from the amount of strain that each individual spring experiences. Therefore, to relate the total strain of the network to that of individual springs, we assume that all the springs experience the same strain in the direction of the applied force as that of the total network and instead modify their stiffnesses, $G_{i}$ 's, such that the total reaction force becomes equal to the applied external force. Thus, the complex system of springs in Figure 2 can be reduced to the one shown in Figure 3. A general form of equivalence between the model networks is discussed by Roscoe [39], by resorting to electrical network theory.

The resultant strain, $\gamma_{r}$, of the system under an applied force is a residual or storage deformation that the material exhibits or recovers in the future in a stress-free state. The liquid matrix 


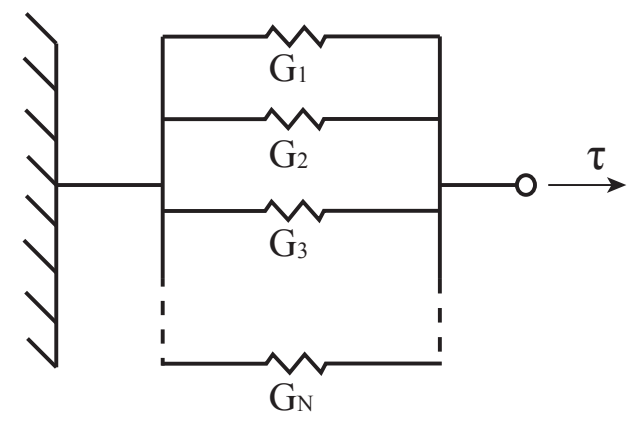

Figure 3: The simplified clay particle network model.

surrounding this particle network provides a viscous contribution to the total stress and is incorporated by adding a dashpot in parallel to the collection of springs. As stress is applied, some connections are destroyed and some are created (this point is more thoroughly explained in the next section). We define the modulus of elasticity, $G_{0}$, as $\sum_{i=1}^{N} \frac{G_{i}}{N}=\frac{G_{0}}{N_{0}}$, where $N$ is the number of current connections and $N_{0}$ is the number of connections of a completely structured material. The resulting clay particle model, depicted in Figure 4, can be written as,

$$
\begin{aligned}
\tau_{d}+\tau_{s_{1}}+\tau_{s_{2}}+\cdots+\tau_{s_{N}} & =\tau \Rightarrow \\
\tau_{d}+\gamma_{r} G_{1}+\gamma_{r} G_{2}+\cdots+\gamma_{r} G_{N} & =\tau \Rightarrow \\
\tau_{d}+\gamma_{r} \frac{N G_{0}}{N_{0}}=\tau_{d}+G_{0} \gamma_{r} \lambda & =\tau,
\end{aligned}
$$

where, the stresses, $\tau$ 's, are defined in Figure 4, and $\lambda=\frac{N}{N_{0}}=\sum_{i=1}^{N} \frac{G_{i}}{G_{0}}$, is the structure parameter with range [0,1]. Furthermore, $\tau_{d}=\mu \dot{\gamma}$, where $\mu$ is the viscosity of the material at $\lambda=0$, and $\dot{\gamma}$ is the strain rate tensor. Therefore, the resulting constitutive model is,

$$
\tau=\mu \dot{\gamma}+\gamma_{r} \frac{N G_{0}}{N_{0}}=\tau_{d}+G_{0} \gamma_{r} \lambda
$$

\subsection{Structure Model}

Experiments have shown that stress and strain controlled measurements performed on claywater suspensions result in different flow curves. In their work on lubricating greases Mas et al. [40] observed that the flow curves obtained from strain controlled tests showed a minimum stress. The same flow curves obtained using stress controlled tests however, did not show such a minimum. Nonetheless, the failure which occurs within the structure of clay-water suspensions when yielding is the same for the two types of test. This signifies that the structure parameter cannot be a function of only stress or only strain but rather a combination of both that is independent of the type of test that is performed. In this respect energy is a viable choice. Therefore, following the work of Moore [14] and Yziquel et al. [27] the time rate of change of the structure parameter is written as, 

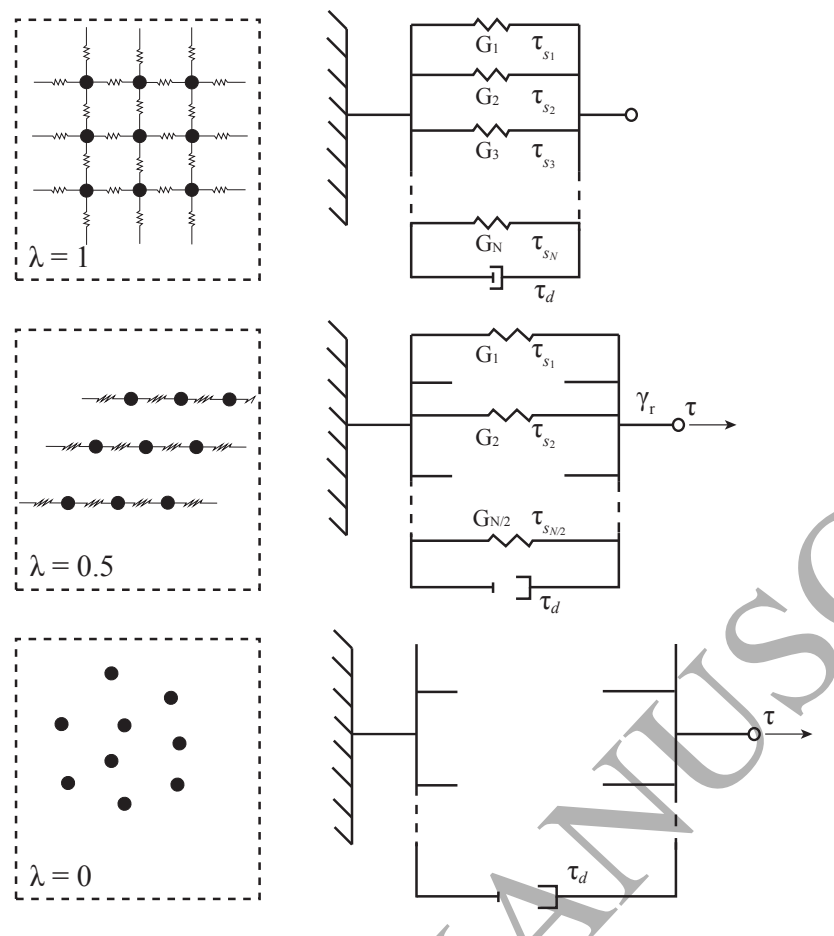

Figure 4: The simplified clay particle network model with complete structure $(\lambda=1)$, top, with half the structure $(\lambda=0.5)$, middle, and with no structure $(\lambda=0)$, bottom.

$$
\frac{d \lambda}{d t}=\alpha(1-\lambda)-|\tau: \dot{\gamma}| \beta \lambda
$$

The first term on the right hand side of (2) represents generation of structure by the Brownian motion. The second term, $|\tau: \dot{\gamma}|$, captures the change in the internal energy of a fluid volume due to the application of a stress or strain rate [41]. Once a stress or a strain rate is applied to the suspension, it damages the structure (i.e., breaks some connections). This damage can also be seen in the increase in the internal energy of the system. Therefore, the increase in the internal energy is used to indicate destruction of structure. Note that equation (2) is symmetric with respect to $\dot{\gamma}$ and $\tau$. In (2), $\alpha$ and $\beta$ are empirical parameters which should be determined through experiments.

\subsection{Residual Strain Model}

Let $\sum_{i=1}^{N} \tilde{\gamma}_{i}(t) \tilde{G}_{i}$ represent the force applied to a clay particle network with $N$ number of connections at a given time $t$. Following the dynamics of the system undergoing a deformation with a strain rate $\dot{\gamma}$, at a later time $t+\Delta t$, let us assume that $M$ number of connections remain, $D$ number of connections are destroyed, and $C$ number of connections are newly created. The strain of the remaining, $N-D=M$, connections at $t+\Delta t$ can be written as,

$$
\gamma(t+\Delta t) \approx \dot{\gamma} \Delta t+\tilde{\gamma}(t),
$$


153 which results in the following expression for the force applied to the clay particle network at time ${ }_{154} t+\Delta t$

$$
\sum_{i=1}^{M}\left(\dot{\gamma} \Delta t+\tilde{\gamma}_{i}(t)\right) \tilde{G}_{i}+\sum_{i=1}^{C} \gamma_{i} G_{i}
$$

155 where, $\gamma_{i}$ and $G_{i}$ are the strains and stiffnesses of the newly created connections, respectively. 156 Furthermore, from the definition of the residual strain one has,

$$
\sum_{i=1}^{N} \tilde{\gamma}_{i}(t) \tilde{G}_{i}=\gamma_{r}(t) \sum_{i=1}^{N} \tilde{G}_{i}=\gamma_{r}(t) \frac{N}{N_{0}} G_{0} \Rightarrow \gamma_{r}(t)=\frac{\sum_{i=1}^{N} \tilde{\gamma}_{i}(t) \tilde{G}_{i}}{\frac{N}{N_{0}} G_{0}} .
$$

${ }_{157}$ Similarly, from (3), for the residual strain $\gamma_{r}(t+\Delta t)$ one has,

$$
\begin{aligned}
\sum_{i=1}^{M}\left(\dot{\gamma} \Delta t+\tilde{\gamma}_{i}(t)\right) \tilde{G}_{i}+\sum_{i=1}^{C} \gamma_{i} G_{i} & =\gamma_{r}(t+\Delta t) \sum_{i=1}^{M+C} \widehat{G}_{i}=\gamma_{r}(t+\Delta t) \frac{M+C}{N_{0}} G_{0} \Rightarrow \\
\gamma_{r}(t+\Delta t) & =\frac{\sum_{i=1}^{M}\left(\dot{\gamma} \Delta t+\tilde{\gamma}_{i}(t)\right) \tilde{G}_{i}+\sum_{i=1}^{C} \gamma_{i} G_{i}}{\frac{M+C}{N_{0}} G_{0}} .
\end{aligned}
$$

Subtracting (4) from (5) and dividing by $\Delta t$ yields,

$$
\begin{aligned}
& \frac{\gamma_{r}(t+\Delta t)-\gamma_{r}(t)}{\Delta t}=\frac{\sum_{i=1}^{M}\left(\dot{\gamma} \Delta t+\tilde{\gamma}_{i}(t)\right) \tilde{G}_{i}+\sum_{i=1}^{C} \gamma_{i} G_{i}}{\frac{M+C}{N_{0}} G_{0} \Delta t}-\frac{\sum_{i=1}^{N} \tilde{\gamma}_{i}(t) \tilde{G}_{i}}{\frac{N}{N_{0}} G_{0} \Delta t} \Rightarrow \ldots \\
\cdots= & \left(\frac{N_{0}}{M+C}\right) \sum_{i=1}^{M} \frac{\dot{\gamma} \tilde{G}_{i}}{G_{0}}+\left(\frac{N_{0}}{M+C}\right) \sum_{i=1}^{C} \frac{\dot{\gamma} G_{i}}{G_{0}}+\left(\frac{M}{M+C}-1\right) \frac{\gamma_{r}(t)}{\Delta t} \Rightarrow \ldots \\
& \frac{\gamma_{r}(t+\Delta t)-\gamma_{r}(t)}{\Delta t}=\dot{\gamma}-\left(\frac{C}{M+C}\right) \frac{\gamma_{r}(t)}{\Delta t} .
\end{aligned}
$$

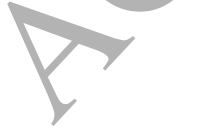

$$
\begin{aligned}
\tau & =G_{0} \lambda \gamma_{r}+\mu \dot{\gamma}, \\
\dot{\lambda} & =\alpha(1-\lambda)-|\tau: \dot{\gamma}| \beta \lambda, \\
\dot{\gamma}_{r} & =\dot{\gamma}-\frac{\alpha(1-\lambda)}{\lambda} \gamma_{r} .
\end{aligned}
$$

${ }_{164}$ Generalization of (8) to (10) for the case of simple shear and vortex flows in three dimensions ${ }_{165}$ is given in Appendix A and Appendix B. 
For stresses $\tau_{c}<\tau<\tau_{y}$, if the material is flowing, it will end up with a structure $\lambda_{e}=\lambda_{e,-}<1$. 185 However, if the material has not disintegrated, i.e., $\lambda_{e}<\lambda_{e,+}$, then it will end up at $\lambda_{e, y}=1$.

\subsection{Evolution of the structure parameter, $\lambda$, for various values of stress}

In equilibrium, the number of connections created equals the number of connections destroyed, therefore, $\dot{\lambda}=0 \Rightarrow \lambda_{e}=\alpha /(\alpha+\beta|\tau: \dot{\gamma}|)$ and the rate of change of residual strain is zero, i.e., $\dot{\gamma}_{r}=0$. Therefore,

$$
\frac{d \gamma_{r}}{d t}=\dot{\gamma}-\frac{\gamma_{r} \alpha\left(1-\lambda_{e}\right)}{\lambda_{e}}=0 \Rightarrow \gamma_{r}=\frac{\lambda_{e} \dot{\gamma}}{\alpha\left(1-\lambda_{e}\right)} .
$$

Inserting (11) in (8) yields the effective viscosity,

$$
\tau=\left(\frac{G_{0} \lambda_{e}^{2}}{\alpha\left(1-\lambda_{e}\right)}+\mu\right) \dot{\gamma} \Rightarrow \mu_{e}\left(\lambda_{e}\right)=\frac{G_{0} \lambda_{e}^{2}}{\alpha\left(1-\lambda_{e}\right)}+\mu .
$$

Hence,

$$
\dot{\gamma}=\frac{\tau \alpha\left(1-\lambda_{e}\right)}{G_{0} \lambda_{e}^{2}+\mu \alpha\left(1-\lambda_{e}\right)} .
$$

Inserting (13) in (9) yields,

$$
\frac{d \lambda}{d t}=\alpha\left(1-\lambda_{e}\right)-\frac{\tau^{2} \alpha\left(1-\lambda_{e}\right)}{G_{0} \lambda_{e}^{2}+\mu \alpha\left(1-\lambda_{e}\right)} \beta \lambda_{e}=0,
$$

which results in,

$$
\alpha\left(1-\lambda_{e}\right)\left[1-\frac{\tau^{2} \beta \lambda_{e}}{G_{0} \lambda_{e}^{2}+\mu \alpha\left(1-\lambda_{e}\right)}\right]=0 .
$$

The roots to (15) are,

$$
\begin{aligned}
& \lambda_{e, y}=1, \text { and, } \\
& \lambda_{e, \pm}=\frac{\mu \alpha+\beta \tau^{2} \pm \sqrt{\left(\mu \alpha+\beta \tau^{2}\right)^{2}-4 G_{0} \mu \alpha}}{2 G_{0}} .
\end{aligned}
$$

Only one solution to (17) exists when, $|\tau|=\sqrt{\left(\sqrt{4 G_{0} \mu \alpha}-\mu \alpha\right) / \beta}$. This shear stress is called the critical stress, $\tau_{c}$, and is the stress below which a flowing material comes to a stop.

At yield stress $\tau=\tau_{y}$, two solutions exist for (17), however, one of them coincides with (16). Inserting $\lambda_{e, y}=1$ in (17) yields, $\left|\tau_{y}\right|=\sqrt{G_{0} / \beta}$. The other root, $\lambda_{e,-}$, can be obtained by inserting $\tau_{y}$ back into equation (17). This yields, $\lambda_{e,-}=\mu \alpha / G_{0}$. Once $\tau=\tau_{y}, \lambda_{e,-}$ is the stable solution where the structure will end up (since $\lambda_{e, y}=1$ becomes unstable at this point and the material begins to flow).

Figure 5 depicts the development of the structure parameter, $\lambda$, for various initial structures, $\lambda_{0}$, under different constant stresses. 


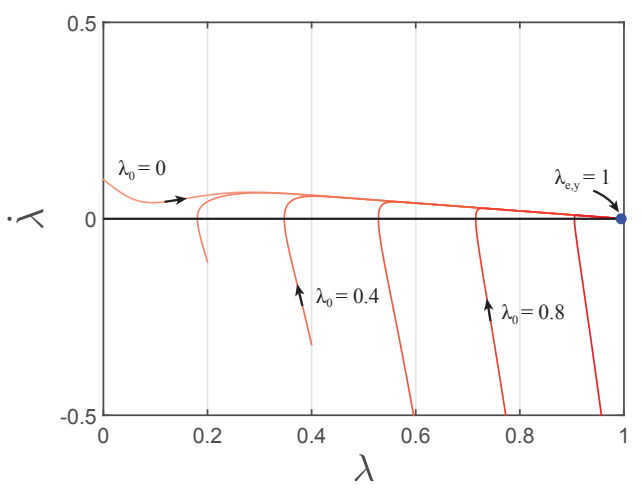

(a) $\tau=\tau_{\mathrm{c}} / 2$

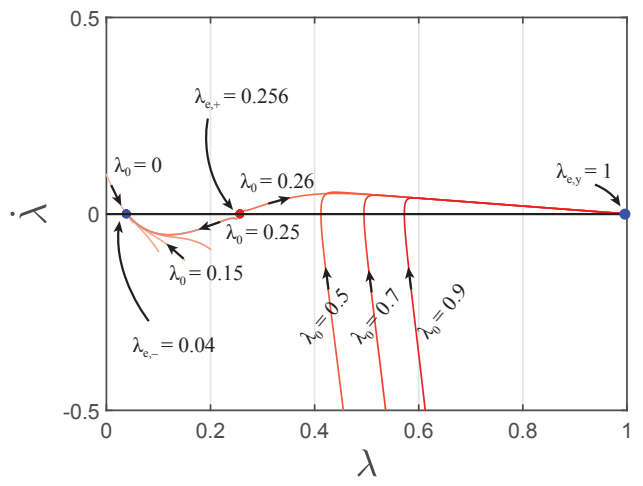

(c) $\tau=3 \tau_{\mathrm{c}} / 2$

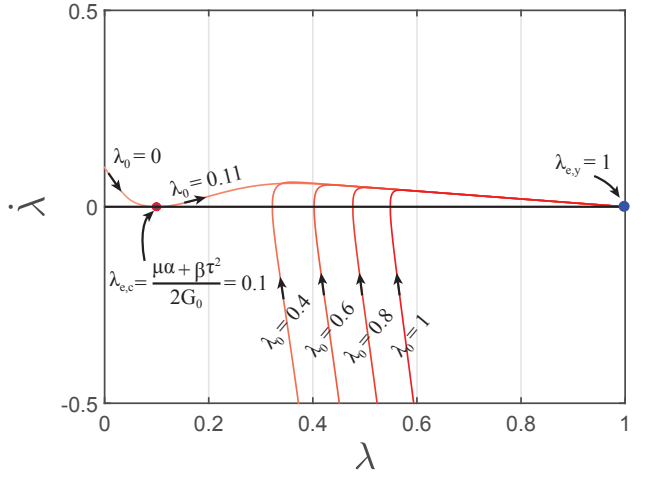

(b) $\tau=\tau_{\mathrm{c}}$

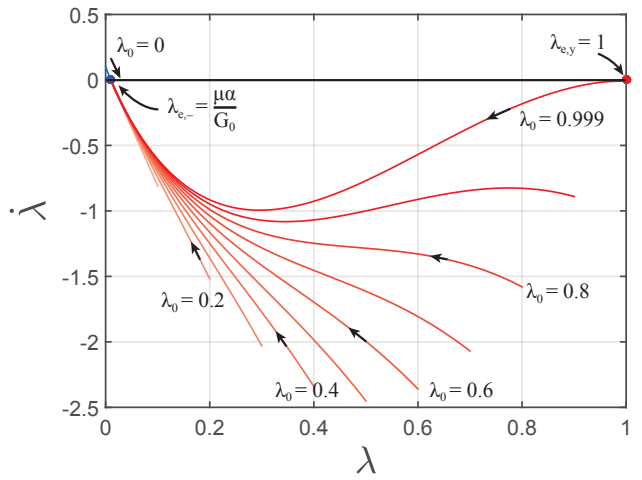

(d) $\tau=\tau_{\mathrm{y}}$

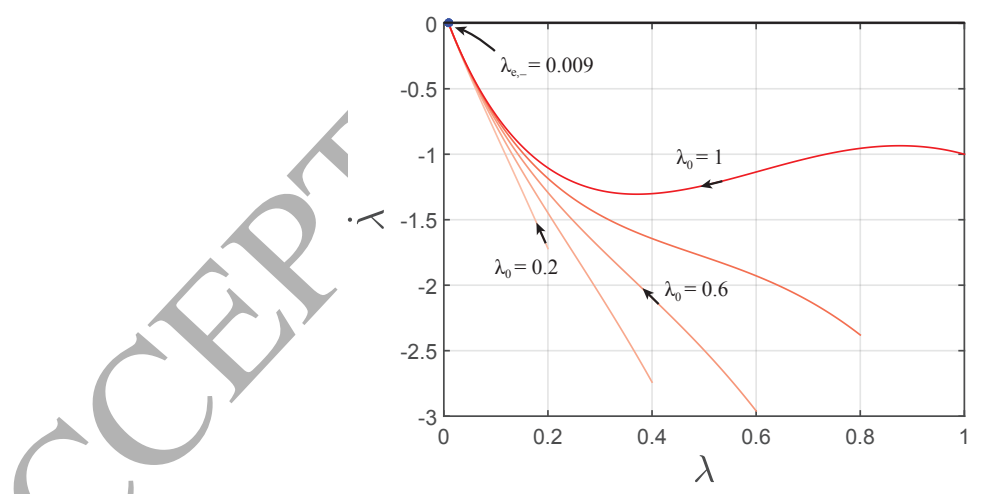

(e) $\tau=1.1 \tau_{\mathrm{y}}$

Figure 5: Change in the structure parameter, $\lambda$, with time, for various initial structures, $\lambda_{0}$, under different stress conditions.

187 2.5. Evolution of the structure parameter, $\lambda$, for various values of strain rate

${ }_{188}$ Following the same procedure as in section 2.4, and writing the stress in terms of the strain 189 rate, equation (15) becomes, 


$$
\left(\beta G_{0} \dot{\gamma}^{2}\right) \lambda_{e}^{3}-\left(\mu \dot{\gamma}^{2} \beta \alpha+\alpha^{2}\right) \lambda_{e}^{2}+\left(\mu \dot{\gamma}^{2} \beta \alpha+2 \alpha^{2}\right) \lambda_{e}-\alpha^{2}=0 .
$$

190

$$
u=\left(\frac{\lambda_{0}-\lambda_{e}}{\lambda_{e}}+e^{(\alpha+\beta|\tau: \dot{\gamma}|) t}\right)^{1-\lambda_{e}}+\left(\frac{\lambda_{0}-\lambda_{e}}{\lambda_{e}}+e^{-(\alpha+\beta|\tau: \dot{\gamma}|) t}\right)^{\lambda_{e}} .
$$



232 regime

${ }_{233}$ For $\tau_{y}=0$ or $\dot{\gamma} \rightarrow \infty$, the model presented in (23) simplifies to the Newtonian fluid model, ${ }_{234} \tau=\mu \dot{\gamma}$. Furthermore, for $\alpha \rightarrow \infty, \tau_{c} \rightarrow \tau_{y}$, and (23) resembles the Bingham elastic model [43].

235 For all the other cases, the rheological model presented by equations (23) to (25) describes a

The complications in solving the integral in (21) can be avoided by introducing a new variable $\zeta=\lambda \gamma_{r}$. The rheological model then reads,

$$
\begin{aligned}
\tau & =G_{0} \zeta+\mu \dot{\gamma}, \\
\dot{\lambda} & =\alpha(1-\lambda)-|\tau: \dot{\gamma}| \beta \lambda, \\
\dot{\zeta} & =\dot{\gamma} \lambda-|\tau: \dot{\gamma}| \beta \zeta .
\end{aligned}
$$

For constant values of $\dot{\gamma}$ and $|\tau: \dot{\gamma}|$, equation (25) can be solved to obtain,

$$
\zeta=\frac{\dot{\gamma}\left(\lambda_{e}-\lambda_{0}\right)}{\alpha} e^{-(\alpha+\beta|\tau: \dot{\gamma}|) t}+\lambda_{e} \frac{\dot{\gamma}}{\beta|\tau: \dot{\gamma}|}+\left[\left(\gamma_{r 0}+\frac{\dot{\gamma}}{\alpha}\right) \lambda_{0}-\left(\frac{\dot{\gamma}}{\beta|\tau: \dot{\gamma}|}+\frac{\dot{\gamma}}{\alpha}\right) \lambda_{e}\right] e^{-\beta|\tau: \dot{\gamma}| t}
$$

where, $\gamma_{r 0}=\gamma_{r}(t=0)$.

\subsection{Types of flow curves}

Let $f$ be a map sending $\lambda_{e}$ to its corresponding stress $\tau$, i.e., $f\left(\lambda_{e}\right): \lambda_{e} \rightarrow \tau$, and let $g$ be a map sending $\lambda_{e}$ to its corresponding strain rate, $\dot{\gamma}$, i.e., $g\left(\lambda_{e}\right): \lambda_{e} \rightarrow \dot{\gamma}$. Then following the discussion in Sections 2.4 and 2.5, $f$ is non-injective, while $g$ is bijective.

Let us imagine a material with a complete structure, $\lambda=1$, and increase the stress until $\tau=\tau_{y}$. Increasing $\tau$ beyond $\tau_{y}$ results in a jump in the equilibrium structure parameter from $\lambda=1$ to $\lambda_{e,-}$. Since $g$ is bijective, the strain rate also shows a jump at this point from $g(\lambda=1)=\dot{\gamma}_{e, y}$ to $g\left(\lambda_{e,-}\right)=\dot{\gamma}_{e,-}$. This path is shown in Figure 7 by red arrows.

Now imagine a material under a high shear stress and with zero structure, $\lambda=0$, and decrease the stress until $\tau=\tau_{c}$. Decreasing $\tau$ beyond $\tau_{c}$ results in a jump in the equilibrium structure parameter from $\lambda_{e, c}$ to $\lambda=1$. Again, since $g$ is bijective, the strain rate also shows a jump at this point from $g\left(\lambda_{e, c}\right)=\dot{\gamma}_{c}$ to $g(\lambda=1)=\dot{\gamma}_{e, y}$. This path is shown in Figure 7, by black arrows.

Note that in Figure 7, the slope of the initial rise in the flow curve, resembling elastic solid behavior, as well as the stress at the point of yielding, are dependent on the speed at which the stress is increased. This topic will be explained more thoroughly in section 2.8 .

Non-injectivity of $f$, results in a different picture for strain controlled hysteresis plots. For $\tau_{c}<\tau<\tau_{y}$, there are three equilibrium structure parameters (non-injectivity of $f$ ) and each structure parameter corresponds to only one shear rate (bijectivity of $g$ ). Consequently, multiple strain rate values correspond to one specific stress in this regime. Figure 8 shows a strain controlled hysteresis plot. The red and black arrows show the path of increasing and decreasing strain rate, respectively.

Note that the model has the ability to capture the critical yield stress, i.e., the minimum stress in a strain controlled flow curve. For clay-water suspensions, the falling region of the flow curve corresponds to the shear banding instability [9]. The model has an unstable equilibrium solution in this transitional region and therefore, mimics the physical behavior of the material well in this thixotropic yield stress fluid. 


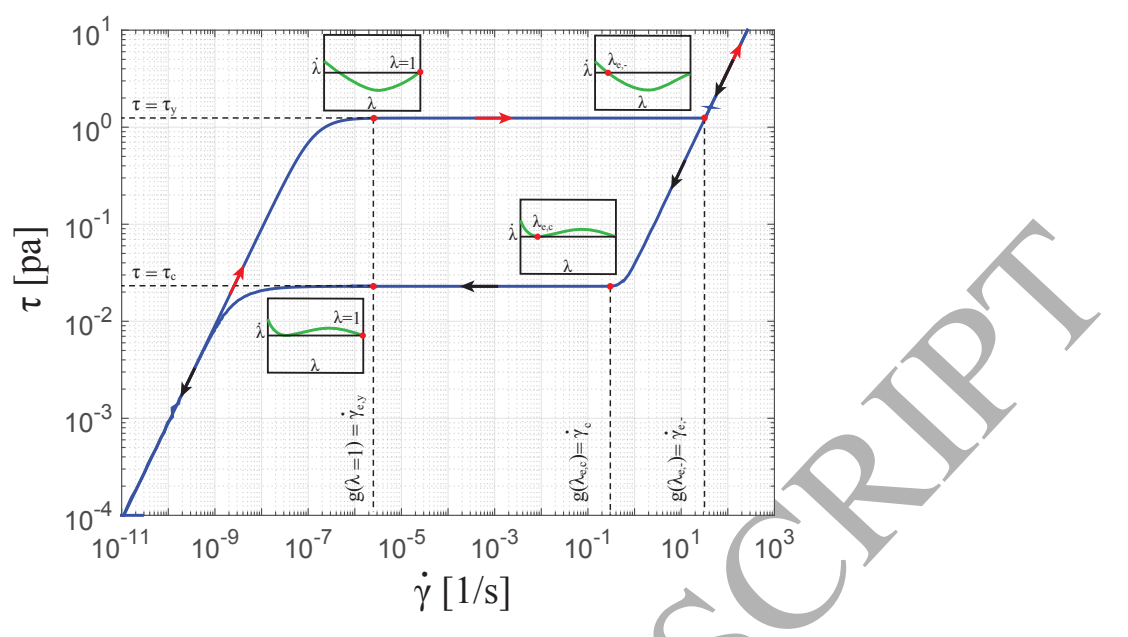

Figure 7: Stress controlled hysteresis plot obtained from equations (23) to (25). The red arrows show the path of increasing stress and the black arrows show the path of decreasing stress. The inset plots depict the bifurcation of the structure parameter.

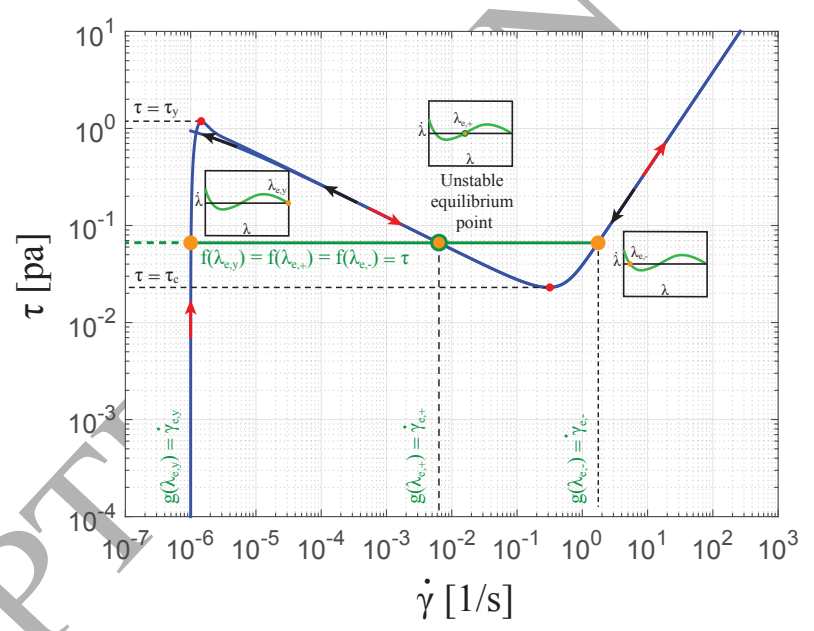

Figure 8: Strain controlled hysteresis plot obtained from equations (23) to (25). The red arrows show the path of increasing strain rate and the black arrows show the path of decreasing strain rate. The inset plots depict the bifurcation of the structure parameter.

$237 \quad$ 2.8. Apparent yield stress

In order to make a stationary material with a structure parameter, $\lambda<1$, flow, one has to overcome the apparent yield stress that corresponds to $\lambda_{e,+}$ (Figure 5), i.e.,

$$
\left|\tau_{y,+}\right|=\sqrt{\frac{G_{0} \lambda_{e,+}^{2}+\mu \alpha\left(1-\lambda_{e,+}\right)}{\beta \lambda_{e,+}}} .
$$


In general, the structure state of a stationary material at a given time is $\lambda_{e,+}$. Therefore, in order to break this structure, a stress level higher than $\left|\tau_{y,+}\right|$, where, $\tau_{c}<\tau_{y,+}<\tau_{y}$, should be imposed on the material. For $\lambda_{e,+}=1, \tau_{y,+}=\tau_{y}$, and for $\lambda_{e,+}=\lambda_{e, c}, \tau_{y,+}=\tau_{c}$ (Figure 5). Once $\tau_{y}<\tau$, there are no longer any unstable equilibrium structure points, $\lambda_{e,+}$, and consequently, there are no apparent yield stresses.

In stress versus strain rate plots, the apparent yield stress is the stress at which the jump in strain rate occurs. To analyze such a case, let $\tau=\tau(t)$, be a logarithmic staircase function with a constant step duration, $\Delta t$, (inset plot of Figure $9(\mathrm{a})$ ). During each step duration, $\Delta t$, equations (23) to (25) are then solved for each constant stress. Figure 9(a) shows the stress versus the strain rate value that is obtained at the end of each time step. Figure 9(b) shows the stress versus the structure parameter in blue and the apparent yield stress, $\tau_{y,+}$, versus $\lambda_{e,+}$ in red, all computed at the end of each time step. The point where the blue and the red curves cross corresponds to the structure state at which $\tau_{y,+}<\tau$, and the material first begins to flow, i.e., $\lambda \rightarrow \lambda_{e,-}$ (Figure 5).

Since from the apparent/transient stress versus strain rate plots (experimental or in this case

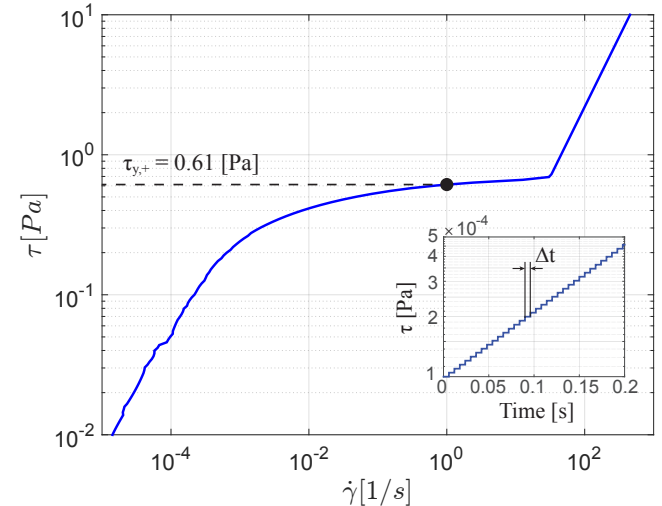

(a) Stress versus strain rate plot.

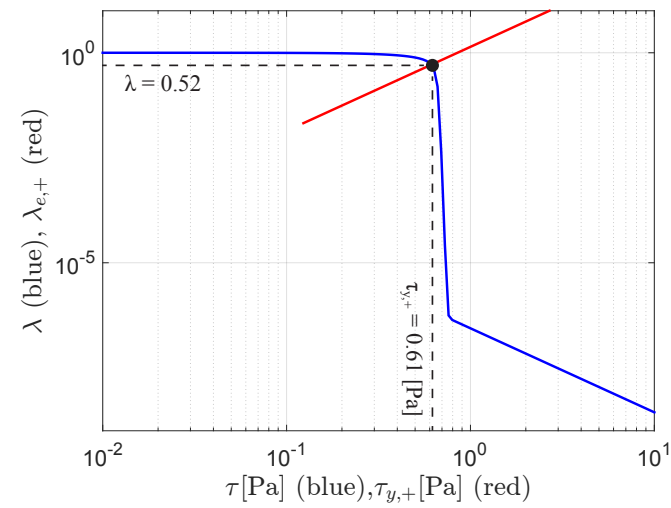

(b) Structure parameter versus stress.

Figure 9: Transient stress versus strain rate plot (a), and structure parameter versus stress curve (b), obtained at the end of each time interval $\Delta t$, for a logarithmic staircase input function shown in the inset plot of (a). The red and blue curves in (b) show the change in $\lambda_{e,+}$ with the apparent yield stress $\tau_{y,+}$, and the structure parameter, $\lambda$, with stress, $\tau$, respectively. 


\begin{tabular}{|c|c|c|c|c|} 
Authors & Const. model & Structure model & Elastic strain model & Emp. parameters \\
\hline $\begin{array}{c}\text { Mujumdar } \\
\text { et al. [33] }\end{array}$ & $\begin{array}{c}\tau=\lambda G \gamma_{e}+ \\
(1-\lambda) K \dot{\gamma}^{n}\end{array}$ & $\dot{\lambda}=k_{1} \dot{\gamma}_{+} \lambda+k_{2}(1-\lambda), \dot{\gamma}_{+}=\left\{\begin{array}{c}0: \dot{\gamma} \gamma_{e} \leq 0, \\
0: \dot{\gamma} \gamma_{e}>0 .\end{array}\right.$ & $\left\{\begin{array}{c}\dot{\gamma}_{e}=\dot{\gamma}:\left|\gamma_{e}\right|<\gamma_{c o} \lambda^{m}, \\
\gamma_{e}=\gamma_{c o} \lambda^{m}:\left|\gamma_{e}\right|>\gamma_{c o} \lambda^{m} .\end{array}\right.$ & $\begin{array}{c}G, n, K, k_{1}, \\
k_{2}, \gamma_{c o}, m\end{array}$ \\
\hline $\begin{array}{c}\text { Dullaert and } \\
\text { Mewis [34] }\end{array}$ & $\begin{array}{c}\tau=\lambda G_{0} \gamma_{e}+ \\
\lambda \eta_{s t, 0} \dot{\gamma}+\eta_{\infty} \dot{\gamma}\end{array}$ & $\dot{\lambda}=\left(1 / t^{\beta}\right)\left(-k_{1} \dot{\gamma} \lambda+k_{2} \dot{\gamma}^{0.5}(1-\lambda)+k_{3}(1-\lambda)\right)$ & $\dot{\gamma}_{e}=(1 / t)^{\beta}\left(\tau \gamma_{c}-\tau_{s s} \gamma_{e}\right)$ & $\begin{array}{c}\gamma_{c}, G_{0}, \eta_{s t, 0}, \eta_{\infty}, \\
\beta, k_{1}, k_{2}, k_{3}\end{array}$ \\
\hline This Study & $\begin{array}{c}\tau=\lambda G_{0} \gamma_{r}+ \\
\mu \dot{\gamma}\end{array}$ & $\dot{\lambda}=-\beta|\tau: \dot{\gamma}| \lambda+\alpha(1-\lambda)$ & $\dot{\gamma}_{r}=\dot{\gamma}-\frac{\gamma_{r} \alpha(1-\lambda)}{\lambda}$ & $\mu, G_{0}, \alpha, \beta$
\end{tabular}

Table 1: The structural kinetic models of Mujumdar et al. [33], Dullaert and Mewis [34], and this study.

\subsubsection{Comparison to the model of Mujumdar et al. [33]}

In [33], Mujumdar et al. present a model based on the network association theory where the material is viewed as a network of small indivisible particles. Once shear is applied to the material, the network breaks down into flocs. If breakdown is continued, the flocs eventually decompose into individual particles. In their formulation, the time-dependent elastic limit of the material is related to the size of the flocs via the structure parameter and an exponent, $m$, that characterizes the elastic limit of the flocs.

It is difficult to express the phenomenology that is presented in [33] (regarding the breakdown of the network into flocs and ultimately into particles) with a picture, such as the one shown in Figure 4. Similar to [33], the model that is presented in the current study is based on the network theory. However, unlike the model of Mujumdar et al., here it is assume that the behavior of the material is uniform and scale invariant. This precludes the need for an exponent such as $m$. A key feature of the model of Mujumdar et al. is a smooth transition from an elastically dominated response to a viscous response, with no discontinuity in the stress-strain curve (Figure $10(\mathrm{~b})$ ). Nonetheless, the formulation of both the structure parameter and the elastic strain are discontinuous in their work, which makes numerical computation cumbersome (Table 1).

Analogous to [33], the model presented here allows for a smooth transition from an elastically dominated response to a viscous response, with no discontinuity in the stress-strain curve (Figure 10(a)). However, in contrast to [33], the formulations of both, the structure parameter and the elastic strain, are continuous in the model presented here.

Following the same procedure as in section 2.4 , the evolution of the structure parameter under various stress conditions can be eyaluated for the model in [33]. To allow comparison with the current study, let the parameter $n=1$ (no shear thinning) in Table 1 . Then, in equilibrium, the following expression can be derived for the structure parameter,

$$
k_{1} G \gamma_{c o} \lambda_{e}^{m+1}+K k_{2} \lambda_{e}^{2}-2 K k_{2} \lambda_{e}+K k_{2}-k_{1} \tau=0 .
$$

It can be seen that depending on the value of the exponent, $m$, equation (28) can have different numbers of roots. For instance for $m=-0.3$ (a representative case from [33]), this equation has in total 20 roots, repeated, as well as distinct (this can be shown by a change of variable from $\lambda_{e}$ to $Q$, where $Q^{10}=\lambda_{e}$ ). This suggests that the model of Mujumdar et al. may be difficult to interpret with regards to the phenomenon of viscosity bifurcation.

Figure 11 shows the strain controlled flow curves obtained from the model of Mujumdar et al. [33] (blue), and from the current study (red). It can be seen that qualitatively, the two models result in very similar flow curves. Nonetheless, there are small discrepancies that make the differences between the two models more conspicuous. A $C^{1}$ discontinuity can be observed within the non-linear elastic regime of the blue curve. This is not the case for the flow curve obtained from the current study. Furthermore, the two models show slightly different behavior at the end of the liquefaction and the beginning of the viscous regimes. 


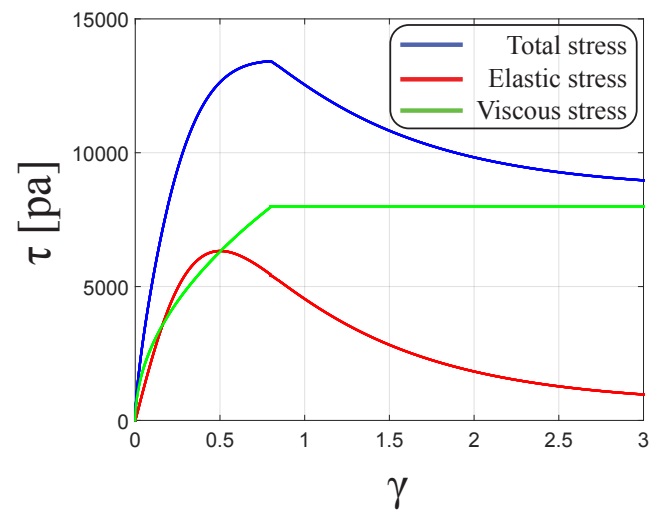

(a) Elastic and viscous contribution to the total stress according to the model from this study.

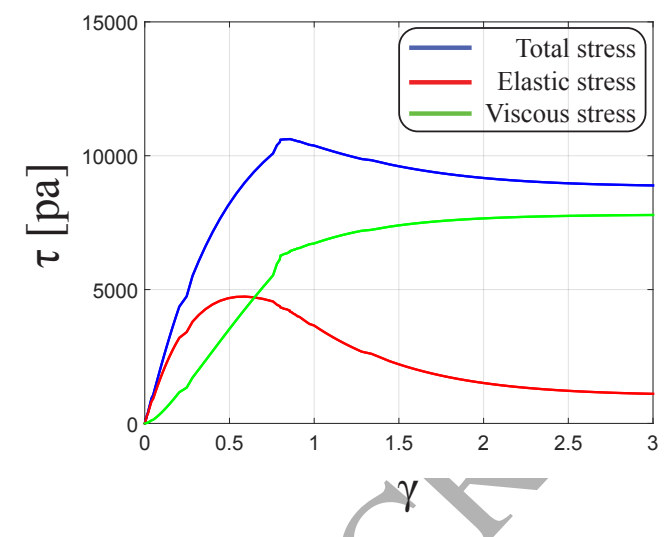

(b) Elastic and viscous contribution to the total stress according to the model of Mujumdar et al.

Figure 10: Prediction of the elastic, viscous, and total stress response from the model presented in the current study (a), and from the model presented by Mujumdar et al. [33] (b), during the start-up of a steady shear flow, $\dot{\gamma}=20 \mathrm{~s}^{-1}$. The parameters used in the model from this study are: $G_{0}=25000 \mathrm{~Pa}, \alpha=1 \mathrm{~s}^{-1}, \mu=400 \mathrm{~Pa} \cdot s, \beta=0.00016 \mathrm{~Pa}^{-1}$. The parameters used in the model of Mujumdar et al. are: $G=25000 \mathrm{~Pa}, K=400 \mathrm{~Pa} \cdot \mathrm{s}, k_{1}=2, k_{2}=1 \mathrm{~s}^{-1}, \gamma_{c o}=$ $0.5, n=1, m=-0.33$.

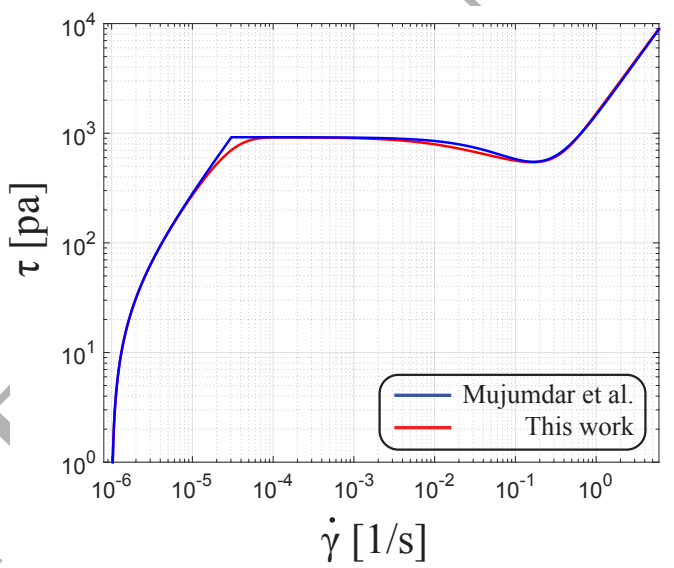

Figure 11: Strain controlled flow curves obtained from the model of Mujumdar et al. [33] (blue), and from the current study (red). The parameters used to obtain the blue curve are: $G=40000 \mathrm{~Pa}, \mathrm{~K}=1500 \mathrm{~Pa} \cdot \mathrm{s}, \mathrm{k}_{1}=10, k_{2}=$ $1 s^{-1}, \gamma_{c o}=0.023, n=1, m=0.15$. The parameters used to obtain the red curve are: $G_{0}=40000 P a, \alpha=1 s^{-1}, \mu=$ $1500 \mathrm{~Pa} \cdot s, \beta=0.04 \mathrm{~Pa}^{-1}$.

Finally, the model presented by Mujumdar et al. contains six empirical parameters (not including $n$ ). In contrast, the model presented in this study has four parameters.

\subsubsection{Comparison to the model of Dullaert and Mewis [34]}

In [34], Dullaert and Mewis present a general structural kinetic model to describe the flow behavior of thixotropic systems. Analogous to the current study, in their work the total stress is divided into a structure-dependent elastic and a viscous contribution. 
To describe the elastic contribution, Dullaert and Mewis propose a single stress-dependent kinetic equation that allows the aggregates to relax after a reduction in hydrodynamic stress and stretch when the stress is increased. This is in contrast to the approach taken in the current study where, the kinetic equation for the elastic strain of the flowing structure is derived from the first principles using the structure model, and based on a set of physical assumptions regarding particle interactions at the micro-scale.

Dullaert and Mewis state that the model in [34] can predict overshoot stresses that are larger than the apparent yield stress during start up flows. The model presented herein however, does not predict such overshoot stresses. According to the model in the current study, as soon as shear is applied to the material, the structure begins to break down. Depending on the strain rate, the structure can break down faster (higher strain rates), or slower (lower strain rates), which in turn can cause the material to reach its yield point quickly, or slowly. The stress at the point of yielding is here referred to as the apparent yield stress and is always smaller than the true yield stress of the material. During start up flows, the stress can quickly reach the apparent yield stress (stress over-shoot) and subsequently drop to the stress of the flowing material (Section 4.2). However, it can never exceed the apparent yield stress of the material.

According to Dullaert and Mewis, upon cessation of flow, the model in [34] can predict non-zero values for the stress. The model presented in this study also has this attribute (Section 4.1). The structure model in [34] is not symmetric with respect to the stress and the strain rate. The structure model employed in this study however, is symmetric.

Following the same procedure as in section 2.4, the evolution of the structure parameter under various stress conditions can be evaluated for the model in [34]. This results in the following expression for the structure parameter in equilibrium,

$$
-k_{1}\left(\frac{\tau-G_{0} \lambda_{e} \gamma_{c}}{\eta_{s t, 0} \lambda_{e}+\eta}\right) \lambda_{e}+k_{2}\left(\frac{\tau-G_{0} \lambda_{e} \gamma_{c}}{\eta_{s t, 0} \lambda_{e}+\eta}\right)^{1 / 2}\left(1-\lambda_{e}\right)+k_{3}\left(1-\lambda_{e}\right)=0 .
$$

The roots of this expression are difficult to obtain. This suggests that the model of Dullaert and Mewis may be difficult to interpret with regards to the phenomenon of viscosity bifurcation.

Figure 12 shows the strain controlled flow curves obtained from the model of Dullaert and Mewis [34] (blue), and from the current study (red). It can be seen that qualitatively, the two models result in quite different flow curves. The red curve is non-monotonic and has a minimum. While the blue curve is a monotonically increasing flow curve. The two curves predict very different material behaviors within the non-linear elastic and the liquefaction regimes. However, some of these differences may be attributed to the values of the empirical parameters used here.

Finally, the model presented by Dullaert and Mewis contains eight empirical parameters. In contrast, the model presented in this study has four parameters.

\section{Methodology for obtaining the empirical parameters and the rheometric validation tests}

In this section the methodology behind the stress and strain controlled measurements which, (1) can be used to obtain the empirical parameters, $\mu, G_{0}, \tau_{y}$, and $\alpha$ (note that $\beta=G_{0} / \tau_{y}^{2}$ ), and (2) are used to evaluate the performance of the model, are explained.

The stress controlled shearing conditions within the boundary layer of sediment gravity flows are simulated using stress controlled stress versus strain rate curves. The stress controlled shearing conditions within the boundary layer eddies are simulated using oscillatory stress controlled amplitude sweep tests. The strain controlled shearing conditions within the free shear and boundary 


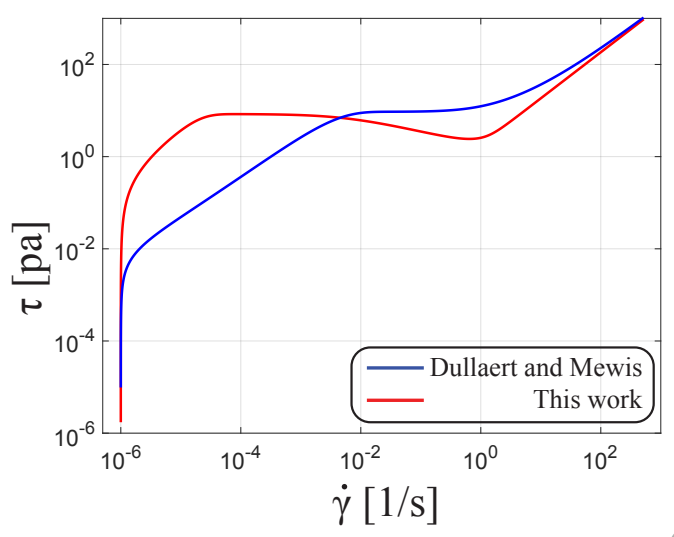

Figure 12: Strain controlled flow curves obtained from the model of Dullaert and Mewis [34] (blue), and from the current study (red). The parameters used to obtain the blue curve are: $\gamma_{c}=0.015, G_{0}=560 P a, \eta_{s t, 0}=8.6 P a \cdot s, \eta_{\infty}=$ $1.84 \mathrm{~Pa} \cdot s, \beta=0.37, k_{1} / k_{3}=0.99 s, k_{2} / k_{3}=0.42 s^{0.5}, k_{3}=0.20 s^{\beta-1}$. The parameters used to obtain the red curve are: $G_{0}=560 \mathrm{~Pa}, \alpha=0.55 \mathrm{~s}^{-1}, \mu=1.84 \mathrm{~Pa} \cdot s, \beta=7.9 \mathrm{~Pa}^{-1}$.

layers are simulated using strain controlled stress versus strain rate curves. For the computations, equations (23) to (25) are solved numerically using a variable order backward differentiation formula [44].

\subsection{Mixture preparation and measurement considerations and apparatus}

Mixture of quartz sand from Sibelco with median diameter of $150 \mu \mathrm{m}$ and Crown Kaolinite clay from ActiveMinerals International, with median diameter of $0.18 \mu \mathrm{m}$ were prepared. The sediment volume concentration was varied between $9 \%, 12.65 \%, 15 \%, 17.82 \%$, and $21 \%$ and contained $2 / 3$ sand and $1 / 3$ clay. For the dilute case of $9 \%$ sediment concentration, the suspension is close to the gelling concentration and the low stress measurements may be influenced by artifacts such as surface tension or the shape of the sample periphery. Therefore, the data for this case is not included during apparent yield stress or shear modulus fitting (Figure 14).

MRC302 Anton Paar rheometer was used for all the measurements. To reduce the effect of settling on the measurements, a concentric cylinder geometry was used. The inner cylinder diameter was $28.92 \mathrm{~mm}$. In order to avoid wall slip a sandblasted bob with a diameter of $26.663 \mathrm{~mm}$ and a surface roughness of 4-7 $\mu \mathrm{m}$ was used. The mixtures were introduced inside the cylinder with a syringe. A cover was used during the tests to reduce water evaporation. The measurements were performed at a temperature of $20^{\circ} \mathrm{C}$.

\subsection{Oscillatory stress controlled amplitude sweep tests and shear modulus, $G_{0}$, measurements}

For low values of strain, $\lambda \approx 1 \Rightarrow N \approx N_{0}$, and $G^{\prime} \approx G_{0}$. Therefore, the value of $G_{0}$ can be approximated by the value of the storage modulus, $G^{\prime}$, obtained from amplitude sweep tests at low values of strain.

Prior to the measurements, the samples were left at rest for a period of 3000 seconds in order to regain structure. Subsequently, stress controlled amplitude sweep tests were performed on the samples. The stress amplitude was increased from $0.0001 \mathrm{~Pa}$ to various final values depending on the sediment concentration, and the angular frequency was set to $10 \mathrm{rad} / \mathrm{s}$. 
Figure 13 depicts the storage, $G^{\prime}$, and loss, $G^{\prime \prime}$, moduli as a function of strain for a representative sample with $12.65 \%$ sediment concentration. The dashed line represents the value of $G_{0}$ approximated by the value of $G^{\prime}$ from the linear part of stress controlled amplitude sweep plots. Similar plots were obtained for other sediment concentrations.

Figure 14(a) depicts the shear modulus, $G_{0}$, obtained for various sediment concentration after a rest period of 3000 seconds. The dashed line in Figure 14(a) was obtained by fitting a power law function to the measurement data. The data for the $9 \%$ sediment concentration was not considered during fitting (section 3.1). The resulting $R^{2}$ goodness of fit value of this power law function is 0.99 .
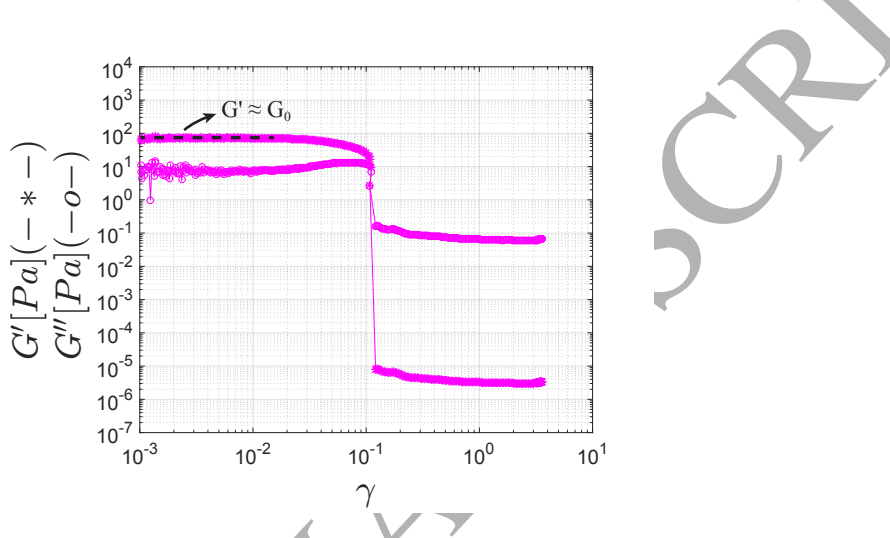

Figure 13: Storage and loss moduli versus strain obtained from stress controlled amplitude sweep test for a sample with $12.65 \%$ sediment concentration, after a resting period of 3000 seconds. The dashed line represents the approximated value of elastic modulus, $G_{0}$.

\subsection{Yield stress, $\tau_{y}$, and viscosity, $\mu$, measurements}

The yield stress and the viscosity values of the mixtures can be obtained from stress controlled rheometry tests. Prior to the measurements, the mixtures were poured into the cylinder geometry and were left to rest for 3000 seconds in order to regain structure. Stress was then increased from $0.0001 \mathrm{~Pa}$ to $1.6,8,11,20$, and $25 \mathrm{~Pa}$ for $9 \%, 12.65 \%, 15 \%, 17.82 \%$, and $21 \%$ sediment concentrations, respectively. The duration for obtaining a data point was set to 2 seconds.

Figure 15 depicts the measurement result of a representative sample with $12.65 \%$ concentration for $0.01<\dot{\gamma}$. The yalue of the apparent yield stress, $\tau_{y,+}$, and viscosity, $\mu$, were obtained from the plots (Figure 15). Equations (23) to (25) were then solved for the same input stress parameters as for the measurements. Assuming 3000 seconds was enough time to reach complete structure $(\lambda=1)$, the initial conditions were set to $\lambda_{0}=1$ and $\gamma_{r 0}=0$. The value of $\tau_{y}$ for each concentration was then adjusted such that the value of $\tau_{y,+}$ obtained from the model matched its value from the measurements.

Figure 14 shows the values of the viscosity and the apparent yield stress for different sediment concentrations. Curve fitting was then performed on the results. The apparent yield stress data for the $9 \%$ sediment concentration was not considered during fitting (section 3.1). For the viscosity and the apparent yield stress, power law functions resulted in fits with $R^{2}=0.99$. The value of $\beta$ was then calculated from the model $\left(\tau_{y}\right)$ and the data $\left(G_{0}\right)$ using the relation $\beta=G_{0} / \tau_{y}^{2}$. 


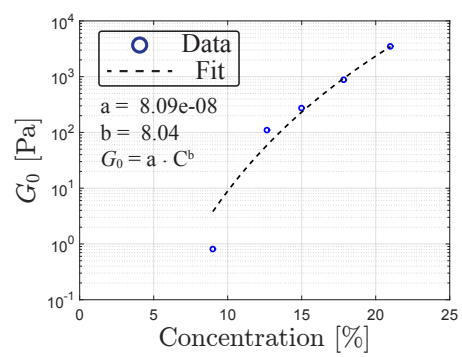

(a) Shear modulus, $\mathrm{G}_{0}$, versus concentration

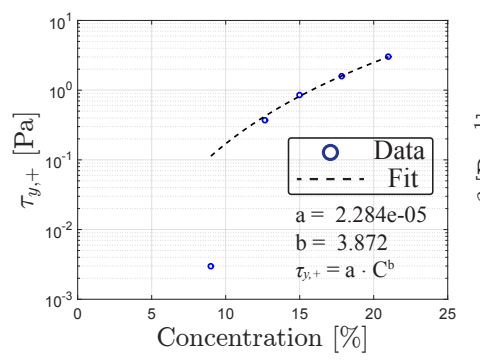

(c) Apparent yield stress versus concentration

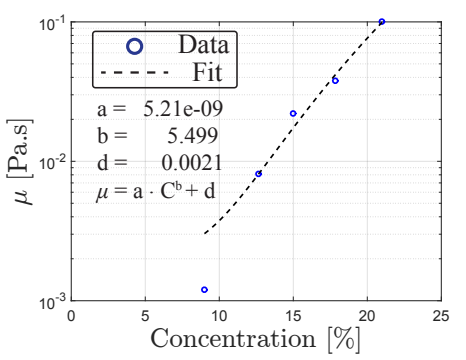

(b) Viscosity versus concentration

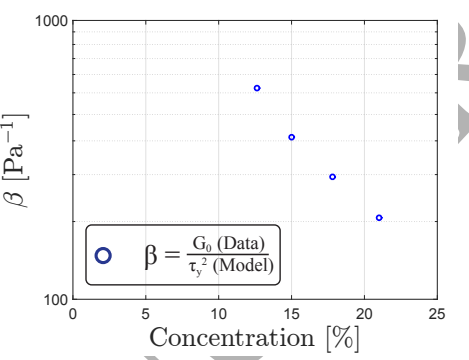

(d) $\beta$ versus concentration

Figure 14: Shear modulus, $G_{0}$, viscosity, $\mu$, apparent yield stress $\tau_{y,+}$, and $\beta$ versus sediment volume concentration. The dashed lines are obtained from curve fitting. For the shear modulus, the viscosity, and the apparent yield stress, power law functions resulted in fits with $R^{2}=0.99$. The apparent yield stress and shear modulus data obtained from samples with $9 \%$ concentration were not considered during fitting.

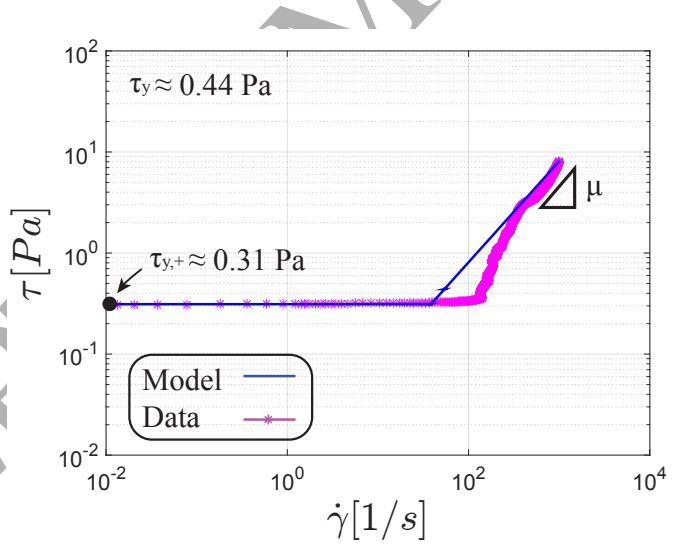

Figure 15: Stress versus strain rate curve obtained from stress controlled measurements after a resting period of 3000 seconds for a representative sample with $12.65 \%$ concentration. The solid blue line is computed using equations (23) to (25).

${ }_{404}$ 3.4. Structure build up rate, $\alpha$

$405 \quad$ From equation (24), for low values of, $|\tau: \dot{\gamma}|$, one has,

$$
\dot{\lambda} \approx \alpha(1-\lambda)
$$


Therefore, in order to obtain the structure build up rate, $\alpha$, the value of $|\tau: \dot{\gamma}|$ should be kept small. Furthermore, the imposed stress amplitude must be set such that it is smaller than the critical stress, $\tau_{c}$.

Immediately after the introduction of the mixture into the cylinder, stress controlled oscillatory tests were performed and the change of storage modulus was monitored in time. The amplitude of the sinusoidal stress input was set to $0.002 \mathrm{~Pa}$ and the angular frequency was set to $0.5 \mathrm{rad} / \mathrm{s}$. Figure 16 shows the change of storage modulus in time obtained from the measurements for samples with $12.65 \%$ and $15 \%$ concentrations.

To quantify the structure build up rate, $\alpha$, equations (23) to (25) were solved. For the computations the same input parameters as the rheometry tests were used. The results were then fitted to the measurement data. The blue dashed lines in Figure 16 depict the results of this procedure. The values of $\alpha$ obtained from this procedure for the samples with $17.82 \%$ and $21 \%$ concentrations were 0.00025 and 0.00015 , respectively.

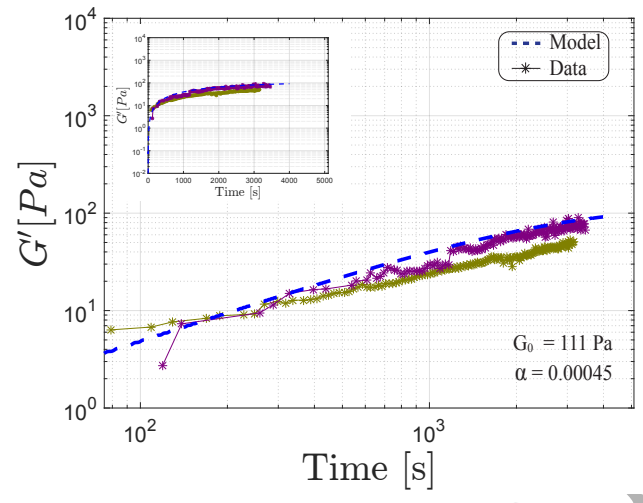

(a) $12.65 \%$ concentration

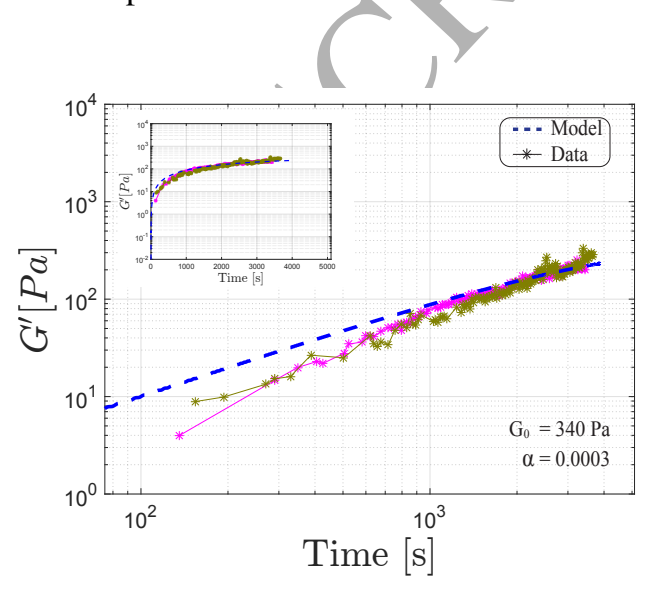

(b) $15 \%$ concentration

Figure 16: Modeled (blue dashed lines) and measured (purple and green lines) storage modulus, $G^{\prime}$, depicting structure build up in time for (a) $12.65 \%$, and (b) $15 \%$ concentrations.

\subsection{Stress controlled measurements}

A set of measurements were performed on a sample with $15 \%$ sediment concentration. In order to enhance the reproducibility of the results, before the measurements, the sample was pre-sheared. This was done by increasing the stress from $0.004 \mathrm{~Pa}$ to $10 \mathrm{~Pa}$, immediately after the introduction of the sample within the cylinder geometry. The duration of this phase was 510 seconds. Next the mixture was left to rest for a period of 9000 seconds to regain structure. Subsequently, the stress was increased ramp logarithmically from $0.004 \mathrm{~Pa}$ to $10 \mathrm{~Pa}$. Each data point was obtained by the rheometer once the shear rate reached a steady state. The mixture was then left to rest for a period of 9000 seconds to regain structure. Finally, a stress controlled oscillatory test was performed on the mixture. The angular frequency was set to $0.5 \mathrm{rad} / \mathrm{s}$ and the stress amplitude was increased ramp logarithmically from $0.004 \mathrm{~Pa}$ to $10 \mathrm{~Pa}$.

For the numerical simulations, the same input conditions as for the measurements were used. For the oscillatory amplitude sweep computations, in order to suppress the noise within the numerical results, a lowpass filter was used in regions before the yielding occurred, and subsequent to yielding, average values of the storage and loss moduli were considered. 

${ }_{473}$ remaining strain is higher. Once the structure is completely recovered, $\gamma_{i}$ converges to a fixed 474 value. 475 All these trends have been discussed by Coussot et al. [35] for various pasty materials.

\subsection{Strain controlled measurements}

Strain controlled measurements were performed on a sample with $15 \%$ sediment concentration. Prior to the measurements, the sample was left at rest for a period of 3000 seconds in order to regain structure. Subsequently, the strain rate was increased ramp logarithmically from $10^{-6} s^{-1}$ to $500 s^{-1}$. The duration for obtaining a data point was set to 2 seconds. The number of measurement points was set to 7830 . Data reproducibility was considered acceptable to not include a pre-shear period. For the numerical simulations, the same input conditions as for the measurements were used.

\section{Results and discussion}

In this section first the solutions of equations (23) to (25) for some selected flows is presented. Subsequently, the ability of the model to reproduce the results obtained from the stress and the strain controlled measurements, discussed in Sections 3.5 and 3.6, is evaluated.

\subsection{Deformation under constant stress}

We would like to investigate the predictions of the model at the solid-liquid transition. To do this we look at, (1) creep tests performed with different stress levels on a completely structured material $\lambda=1$, and (2) creep tests performed with a specific stress level on a material with different initial structure states.

Figure 17(a) depicts material deformation as a function of time for a completely structured material, $\lambda_{0}=1$, under different stress levels, $\tau$, where $\tau(t)=\tau H(t)$, and $H(t)$ is the Heaviside unit step function. As it is common place for pasty materials [35], two regimes can be observed. For stresses smaller than $\tau_{y,+}$, the model predicts an initial rapidly rising phase in the deformation, followed by a leveling off and reaching a plateau phase. For stresses higher than $\tau_{y,+}$, the model predicts a straight line of slope 1 in the logarithmic scale, i.e., the deformation tends to increase at a constant rate. It can be seen that the transition between the two regimes is abrupt in terms of shear rate, i.e., for a smallincrease in stress beyond $\tau_{y,+}$, the slope of $\gamma(t)$ changes abruptly from zero to 1. As it was explained in section 2.8, the value of $\tau_{y,+}$ is a function of how fast the stress ramp is reached and is smaller than $\tau_{y}$ (in this case $\tau_{y,+}=0.5 \tau_{y}$ ).

Figure 17(b) shows the material deformation as a function of time after application of a stress ramp to a material with various initial structure parameters. It can be seen that for an initial structure parameter higher than approximately 0.4 , the material shows an elastic solid behavior. For lower initial structure parameters however, the behavior of the material abruptly transforms to that of a viscous liquid.

Once stress is applied to a material, its structure is damaged and begins to undergo a recovery process known as aging [35]. For very short creep test durations, $t_{w}$, the deformation undergone by the material during the test is almost completely recovered for sufficiently long relaxation times. For longer creep tests on the other hand, the model predicts a drop in $\gamma(t)$ to a remaining value, $\gamma_{i}$. Figure 18 shows the deformation response of a material, as predicted by the model, creep tests with different test durations, $t_{w}$. It can be seen that for longer $t_{w}$, the value of the 


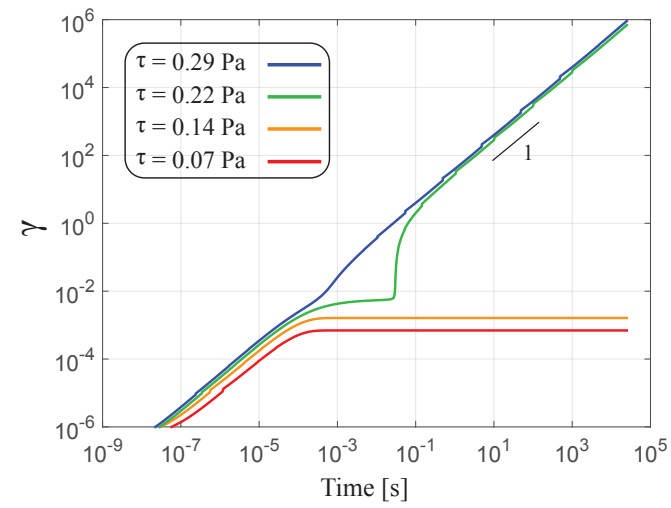

(a) Creep tests under different stresses $\left(\lambda_{0}=1\right)$.

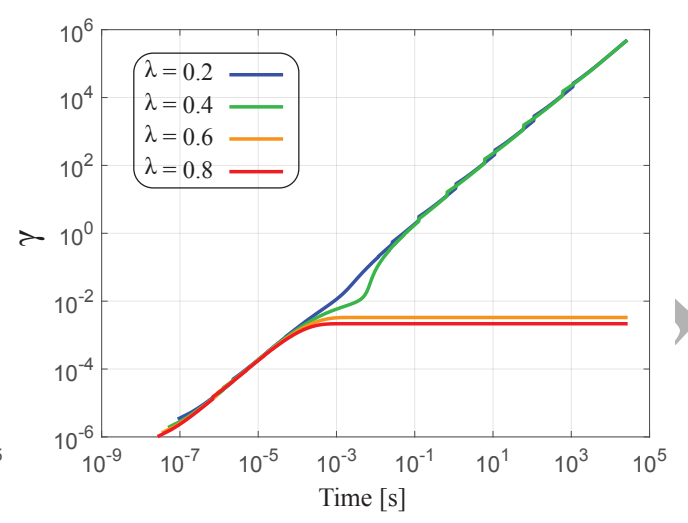

(b) Creep tests for different initial structure parameters $(\tau=0.14 \mathrm{~Pa})$.

Figure 17: Creep test plots obtained from equations (23) to (25) for (a) a completely structured material, $\lambda_{0}=1$, under various stress levels, and (b) for various initial structure states and under a constant stress level, $\tau=0.14 P a$. The value of the yield stress, $\tau_{y}$, was set to $0.37 P a$.

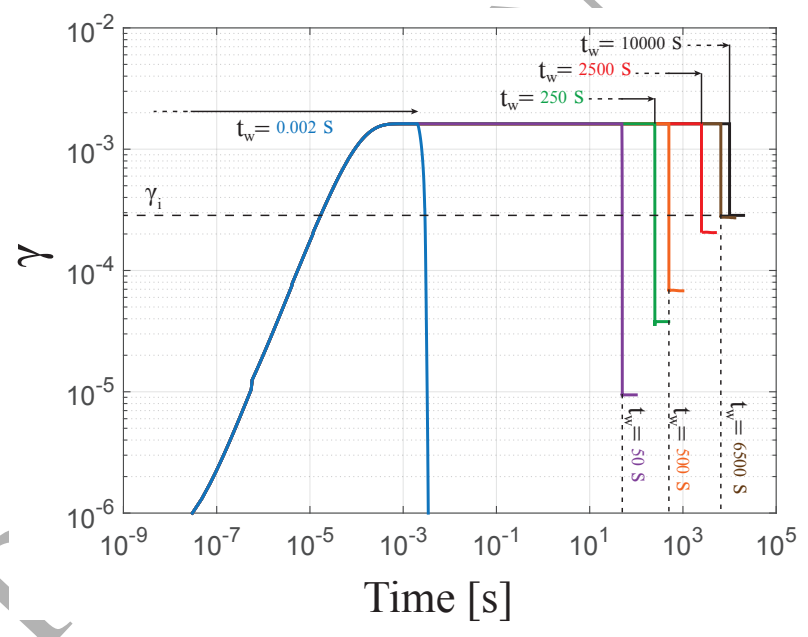

Figure 18. The deformation response of a material obtained from equations (23) to (25) to creep tests with different durations, $t_{w}$. The horizontal dashed line depicts the remaining strain, $\gamma_{i}$, after complete recovery of the material.

\subsection{Deformation under constant strain rate}

Let $\dot{\gamma}(t)=\dot{\gamma}\left(H\left(t-t_{0}\right)-H\left(t-t_{1}\right)+H\left(t-t_{2}\right)\right)$, where $t_{0}$ and $t_{2}$ correspond to the times at which a strain rate of magnitude $\dot{\gamma}$ is applied to a completely structured material, and $t_{1}$ corresponds to 479 the cessation of application of strain rate. The stress response of a material to such an input is ${ }_{480}$ depicted in Figure 19. For small strain rate inputs (Figure 19(a)), the elastic stress is dominant, 481 i.e., $\tau \approx G_{0} \zeta$. In this regime no prominent local maximum can be observed in the stress response ${ }_{482}$ at $t=t_{0}$ (at which point the strain rate $\dot{\gamma}$ is initially applied). Instead, the stress increases until ${ }_{483}$ it reaches a steady value. Once the strain rate is set to zero at $t=t_{1}$, the stress response shows 
no change. This indicates that for $t_{0}<t<t_{1}$, the material undergoes a deformation in this regime that is not recovered once the shear rate is set to zero, i.e., as $\lambda \rightarrow 1$ for long relaxation times, $\gamma_{r}$ approaches a constant value. At $t=t_{2}$, again a strain rate of magnitude $\dot{\gamma}$ is applied to the material. The stress response shows an initial increase at $t=t_{2}$ to a maximum, drops to a minimum, and increases and reaches the same steady value as it had reached just before $t=t_{1}$. This is because the high shear rate after the jump results in a sudden increase in the stress, which then breaks the structure. Since the structure is lost, the stress begins to drop to a minimum (inset plot in Figure 19(a)). At this stage the material begins to recover its structure, and the stress begins to increase and reach a steady state value. The time it takes the material to reach the steady state after $t=t_{2}$ is shorter than the time it took it initially to recover after $t=t_{0}$. This is due to the fact that at $t=t_{2}, \gamma_{r}\left(t_{2}\right)>\gamma_{r}\left(t_{0}\right)=0$. Therefore, it takes a shorter time for $\gamma_{r}$ to reach the steady value, because it is closer to it. The same behavior can be observed in Figure 19(b), with the difference that now at $t=t_{0}$, stress responses show clear local maximums which are larger than the ones at $t=t_{2}$. This signifies that in this regime the applied strain rate is high enough to elicit an elastic response from the material that diffuses for long enough times. The red line in Figure 19(c) marks the initiation of a regime where viscous stresses first become comparable in magnitude to elastic stresses. Both the red and the yellow lines in this figure show a jump at $t=t_{1}$. This is because once the strain rate is set to zero at $t=t_{1}$, the portion of the stress response corresponding to viscous stress vanishes, i.e., $\mu \gamma=0$, and only the elastic part remains. As viscous stresses become the dominant form of stress at high shear rates the jumps in the stress response at $t=t_{1}$ become more severe (Figure 19(d)). Another interesting feature that can be observed from Figure 19 is that for lower strain rates, the time required to reach a steady state increases dramatically [28].

These trends are corroborated by data for various thixotropic materials in the literature [36, 34].

\subsection{Stress controlled measurements}

Figure 20 shows the results of the stress controlled measurements that were described in section 3.5 .

Figure 20(a) presents the stress versus strain rate curves that were obtained from the model (blue line) and the measurements (red circles). The values of the empirical parameters used to solve the equations (23) to (25) are presented in this figure. The inset plot depicts the stress input, corresponding to $\dot{\gamma}>10^{-4} s^{-1}$, that was imposed during the measurements and used during the simulation. It can be seen that the model shows a good agreement with the data within the liquefaction and the fluid regimes.

Figure 20(b) shows the stress as a function of strain. At very low values of strain, i.e., $\gamma<$ $10^{-4}$, the model shows small deviations from the measurements. These deviations did not have the same shape for all the sample measurements and sometime were not present. Due to the complexity of the mixture under investigation, more examination is required to exactly pinpoint the source of this discrepancy. Nonetheless, at low values of strain, i.e., within the linear elastic regime, a relatively good agreement between the data and the model can be observed. At the end of the linear elastic regime and within the non-linear elastic regime, the model again shows some deviations from the data. As before, these deviations were not observed for all the sample measurements and their source may be traced back to the small amounts of slip that may have occurred during the measurement before yielding. The model shows good agreement with the data within the liquefaction and the fluid regimes.

Figure 20(c) shows the measured and the computed values of the storage, $G^{\prime}$, and the loss, $G^{\prime \prime}$, moduli as a function of stress amplitude. A good agreement can be observed between the 


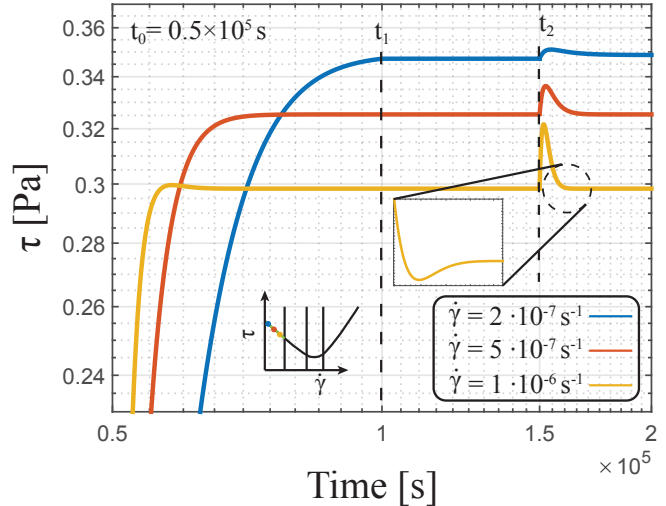

(a) Low strain rate regime

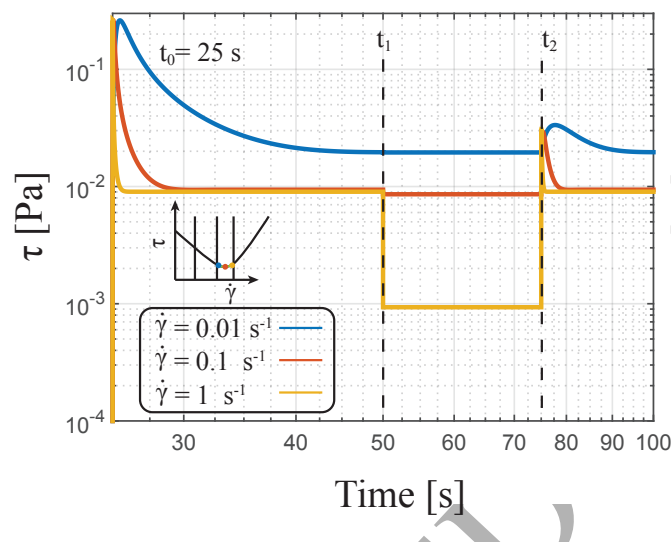

(c) Medium to high strain rate regime

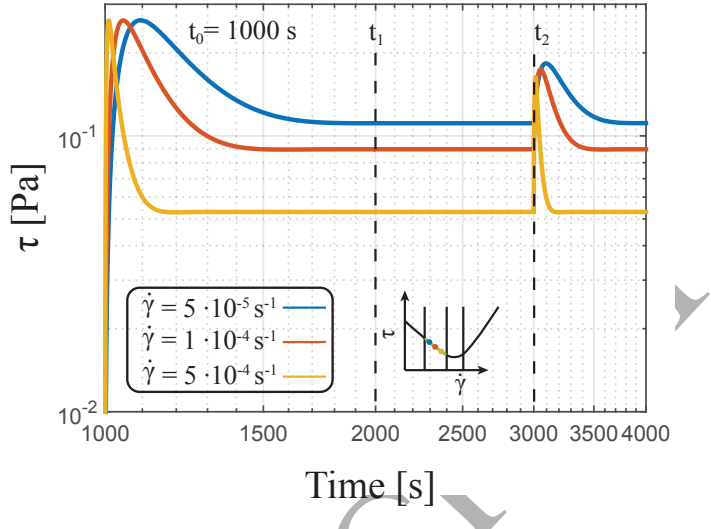

(b) Low to medium strain rate regime

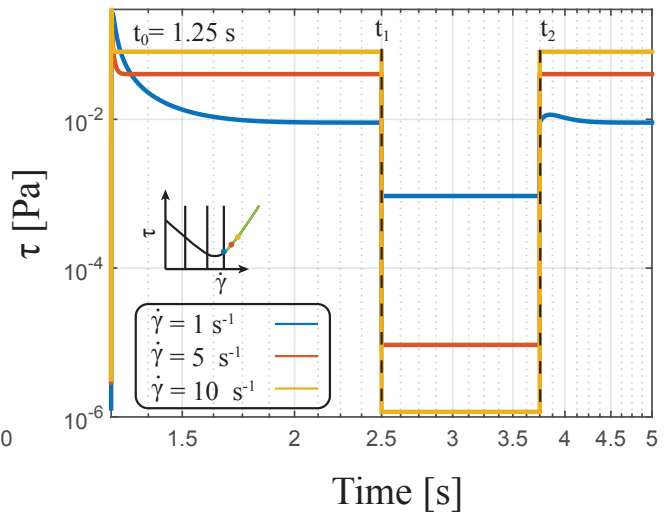

(d) High strain rate regime

Figure 19: The stress response of a material to the strain rate input $\dot{\gamma}(t)=\dot{\gamma}\left(H\left(t-t_{0}\right)-H\left(t-t_{1}\right)+H\left(t-t_{2}\right)\right)$. Since the steady state was achieved faster at higher shear rates, the duration of the input signal (i.e. $t_{2}-t_{0}$ ) was varied for the sake of improved visibility. The inset plot shows a schematic strain controlled flow curve and where the constant strain rate lines may fall on such a plot.

measured and the computed values of the storage and the loss moduli within the linear and the nonlinear elastic regimes. At high stress amplitudes however, the data shows a complex behavior. The model does not capture this behavior and underestimates the average values of the storage and loss moduli within this region. This may be due to the long duration of these tests which may have resulted in water evaporation, and in turn, increased viscosity of the mixture.

\subsection{Transient strain controlled stress versus strain rate curve}

Figure 21 shows the measured (blue stars) and the computed (solid red line) strain controlled shear stress versus strain rate curves for a sample with $15 \%$ concentration. The inset plot depicts a zoomed in view of the flow instability that is associated with the shear banding phenomenon. As discussed by Pignon et al. [9], the shear rate values provided by the rheometer in this region correspond to a very localized or transient flow and are smaller than the actual shear rate values. 


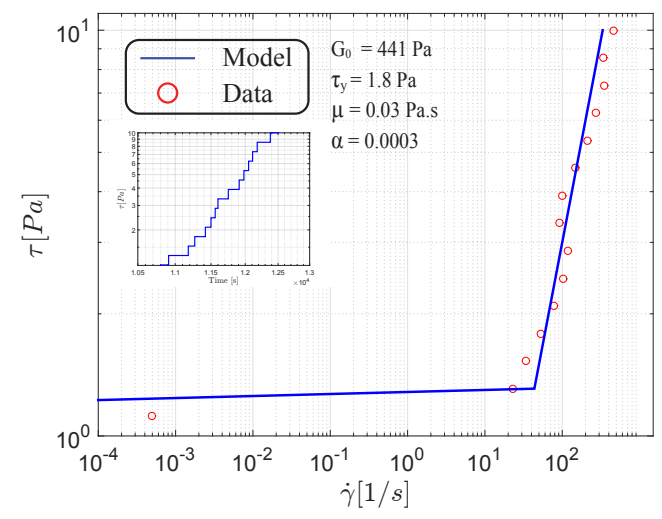

(a) Stress versus strain rate

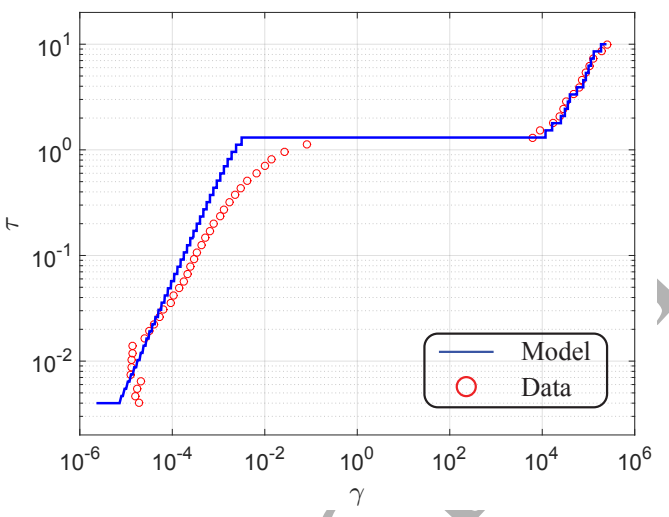

(b) Stress versus strain

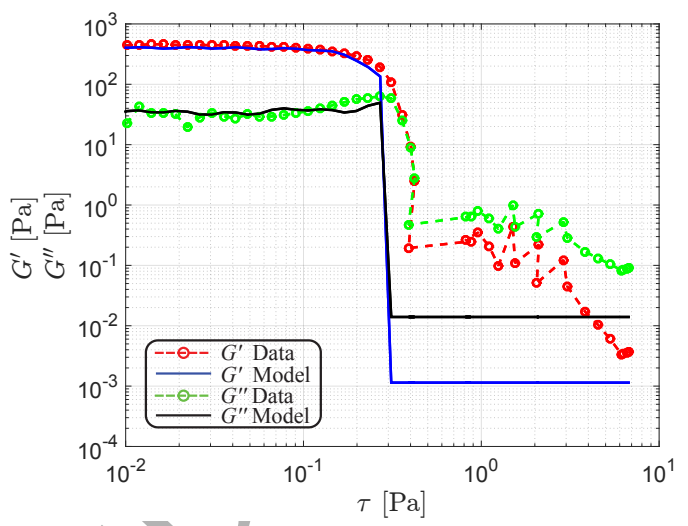

(c) Storage and loss moduli versus stress

Figure 20: Measured (red and green circles) and computed (solid blue and black lines) stress versus strain rate curve (a), stress versus strain curve (b), and storage and loss moduli versus stress curve (c), obtained from stress controlled measurements for $15 \%$ concentration.

Therefore, although with the corrected shear rate values, the qualitative shape of the transient curve would remain the same, the data in this part of the curve should be precluded from comparison with the model.

It can be seen that the measurements and the model both show an initial rise in the shear stress until a maximum yield point followed by a drop to a minimum. The measurements suggest that at an approximate strain rate of $10^{-5} \mathrm{~s}^{-1}$ the mixture begins to exhibit strain hardening. The model fails to capture this phenomenon. At higher strain rates corresponding to $\tau>\tau_{c}$, the measurements and the model begin to again show good agreement.

\section{Conclusions}

A new viscoelastic constitutive relation for modeling of subaqueous clay-rich gravity flows was presented. In order to capture the creep and the yield behavior of the plug layer within 


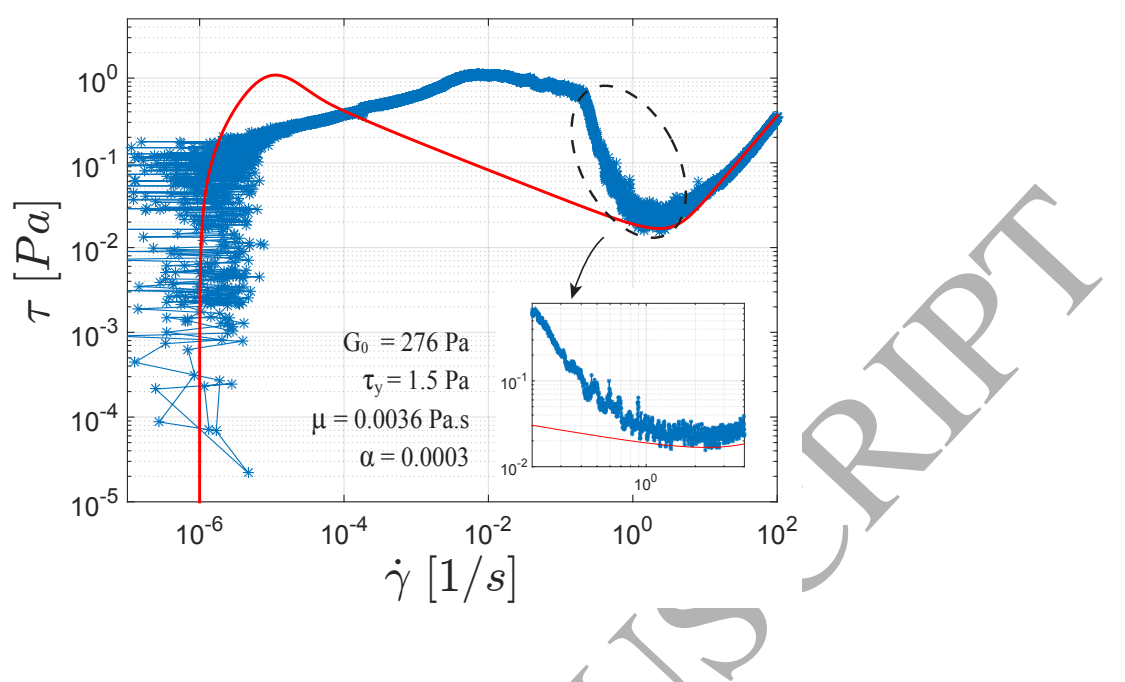

Figure 21: Transient stress versus strain rate curve obtained from strain controlled measurements for a sample with $15 \%$ concentration after a resting period of 3000 seconds (blue stars). The solid red line is computed using equations (23) to (25). The value of the empirical parameters used during the computation are presented inside the plot. The inset plot depicts a zoomed in view of the shear banding instability.

these flows the Kelvin-Voigt model was considered. The total stress is split into an elastic and a viscous part. The importance of the elastic part is determined by the amount of structure. It was explained that for the materials which exhibit a minimum in their strain controlled flow curves the structure parameter must be a symmetric function of the strain rate and the stress. Therefore, the destruction of structure within the material was accounted for using the dissipation energy. An expression for the elastic strain of the flowing structure was then derived.

It was shown that the final set of equations can reproduce the viscosity bifurcation that clay suspensions may exhibit under a given load. This is important for accurate prediction of the run-out distance of gravity flows. The most general flow curves which are allowed by the model were discussed and the response of the model to a constant stress and a constant shear rate inputs was analyzed. It was concluded that the model can reproduce the well documented responses of pasty materials to such tests.

The final set of equations requires four empirical parameters. A methodology was presented for obtaining these parameters. Power law functions were then obtained for their calculations for a limited rest time of 3000 seconds.

The capability of the model was evaluated by comparing its output with the data obtained from stress controlled measurements. At low values of strain, i.e., within the linear elastic regime, a relatively good agreement between the data and the model was observed. At the end of the linear elastic regime and within the non-linear elastic regime, a discrepancy between the model output and the data was observed. This may be due to small amounts of slip that may have occurred before yielding. A good agreement between the model and the measurements was observed within the yielding and the viscous regions of the stress versus strain and the stress versus strain rate curves. For stress controlled oscillatory tests, a good agreement was observed between the measured and the computed values of the storage and loss moduli at low stress amplitudes. At high stress amplitudes on the other hand, the model appears to deviate from 
577 the measurement. This maybe due to the long duration of the tests which may result in water 578 evaporation and in turn increased viscosity of the mixture. For transient strain controlled curves,

579 a good agreement between the measurements and the model was observed at low and high strain rates. The measurements suggest that at intermediate strain rates, the mixture exhibits strain hardening. The model does not capture this phenomenon.

\section{Acknowledgments}

We would like to thank The Netherlands Research Center For Integrated Solid and Earth Science (ISES) for funding this project. We also would like to thank Cor Kasbergen and Dennis den Ouden-van der Horst for insightful comments regarding this work.

\section{Appendix A. Three dimensional formulation of constitutive equation for simple shear flow}

The expression for the total stress tensor, $\sigma$, within a fluid can be written as,

$$
\sigma=p \delta+\tau .
$$

${ }_{588}$ where, $p$ is the thermodynamic pressure, $\delta$ is the unit tensor, and $\tau$ is the stress tensor.

${ }_{589}$ For isotropic fluids in simple shear flow depicted in Figure A.22, the stress tensor can be written 590 as $[45,41]$,

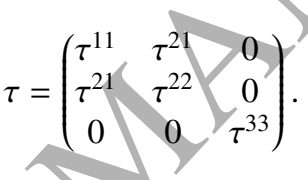

591 The components of the strain tensor can be written as $\gamma^{i j}=\delta-g^{i j}$, where $g^{i j}$ is the inverse of the metric tensor. This yields,

$$
\gamma=\left(\begin{array}{ccc}
-\gamma^{21^{2}} & \gamma^{21} & 0 \\
\gamma^{21} & 0 & 0 \\
0 & 0 & 0
\end{array}\right)
$$

${ }_{593}$ The strain rate tensor can be written as,

$$
\dot{\gamma}=\left(\nabla v+\nabla v^{T}\right)=\left(\begin{array}{ccc}
0 & \dot{\gamma}^{21} & 0 \\
\dot{\gamma}^{21} & 0 & 0 \\
0 & 0 & 0
\end{array}\right) .
$$


594 From the definition of the residual strain and assuming linearity between the stain and the residual 595 strain tensors yields,

$$
\gamma_{r}^{k s}=\frac{G_{i j}^{--k s} \gamma^{i j}}{\lambda G_{0}} .
$$

596 where, the elastic modulus, $G_{i j}^{--k s}$, is a fourth-rank tensor.

597 Assuming a homogeneous and isotropic material yields [45],

$$
\gamma_{r}=\left(\begin{array}{ccc}
\gamma_{r}^{11} & \gamma_{r}^{21} & 0 \\
\gamma_{r}^{21} & \gamma_{r}^{22} & 0 \\
0 & 0 & \gamma_{r}^{22}
\end{array}\right)
$$

${ }_{598}$ Using the fact that the Christoffel symbols, $\Gamma_{k l}^{i}=\frac{1}{2} g^{i m}\left(\frac{\partial g_{m k}}{\partial x_{l}}+\frac{\partial g_{m l}}{\partial x_{k}}-\frac{\partial g_{k l}}{\partial x_{m}}\right)=0$, and taking the 599 time derivative of (A.6) and its basis vectors yields [41],

$$
\dot{\gamma}_{r}=\frac{\partial}{\partial t}\left(\begin{array}{ccc}
\gamma_{r}^{11} & \gamma_{r}^{21} & 0 \\
\gamma_{r}^{21} & \gamma_{r}^{22} & 0 \\
0 & 0 & \gamma_{r}^{22}
\end{array}\right)-\left(\begin{array}{ccc}
2 \dot{\gamma}^{21} \gamma_{r}^{21} & \dot{\gamma}^{21} \gamma_{r}^{22} & 0 \\
\dot{\gamma}^{21} \gamma_{r}^{22} & 0 & 0 \\
0 & 0 & 0
\end{array}\right) .
$$

600 601 simple shear flow can be written as,

$$
\begin{aligned}
\left(\begin{array}{ccc}
\tau^{11} & \tau^{21} & 0 \\
\tau^{21} & \tau^{22} & 0 \\
0 & 0 & \tau^{33}
\end{array}\right) & =G_{0} \lambda\left(\begin{array}{ccc}
\gamma_{r}^{11} & \gamma_{r}^{21} & 0 \\
\gamma_{r}^{21} & \gamma_{r}^{22} & 0 \\
0 & 0 & \gamma_{r}^{22}
\end{array}\right)+\mu\left(\begin{array}{ccc}
0 & \dot{\gamma}^{21} & 0 \\
\dot{\gamma}^{21} & 0 & 0 \\
0 & 0 & 0
\end{array}\right), \\
\dot{\lambda} & =\alpha(1-\lambda)-|\tau: \dot{\gamma}| \beta \lambda, \\
\frac{\partial}{\partial t}\left(\begin{array}{ccc}
\gamma_{r}^{11} & \gamma_{r}^{21} & 0 \\
\gamma_{r}^{21} & \gamma_{r}^{22} & 0 \\
0 & 0 & \gamma_{r}^{22}
\end{array}\right)= & \left(\begin{array}{ccc}
2 \dot{\gamma}^{21} \gamma_{r}^{21} & \dot{\gamma}^{21} \gamma_{r}^{22} & 0 \\
\dot{\gamma}^{21} \gamma_{r}^{22} & 0 & 0 \\
0 & 0 & 0
\end{array}\right)+\left(\begin{array}{ccc}
0 & \dot{\gamma}^{21} & 0 \\
\dot{\gamma}^{21} & 0 & 0 \\
0 & 0 & 0
\end{array}\right) \\
& -\frac{(1-\lambda) \alpha}{\lambda}\left(\begin{array}{ccc}
\gamma_{r}^{11} & \gamma_{r}^{21} & 0 \\
\gamma_{r}^{21} & \gamma_{r}^{22} & 0 \\
0 & 0 & \gamma_{r}^{22}
\end{array}\right) .
\end{aligned}
$$

\section{Appendix B. Three dimensional formulation of constitutive equation for vortex flow}

Within the free shear layer, let us consider a vortex flow in the polar coordinates given by,

$$
\vec{u}=\left(v_{r}, v_{\theta}, v_{z}\right)=\left(0, \frac{C}{r}, 0\right),
$$

604 where, $C$ is a constant and $r$ is the distance from the center of the vortex.

605 Using the rotational symmetry of vortex flow, the stress tensor in polar coordinates can be written 606 as,

$$
\tau=\left(\begin{array}{ccc}
\tau^{11} & 0 & 0 \\
0 & \tau^{11} & 0 \\
0 & 0 & \tau^{33}
\end{array}\right)
$$


The strain tensor can be written as,

$$
\gamma=\left(\begin{array}{ccc}
0 & -\frac{2 C t}{r^{2}} & 0 \\
-\frac{2 C t}{r^{2}} & -\frac{2 C t}{r} & 0 \\
0 & 0 & 0
\end{array}\right)
$$

608 and the strain rate tensor can be written as,

$$
\dot{\gamma}=\left(\begin{array}{ccc}
0 & -\frac{2 C}{r^{2}} & 0 \\
-\frac{2 C}{r^{2}} & 0 & 0 \\
0 & 0 & 0
\end{array}\right)
$$

609 610

611

From the definition of the residual strain and assuming linearity between the stain and the residual strain tensors and a homogeneous, isotropic, material yields,

$$
\gamma_{r}=\left(\begin{array}{ccc}
\gamma_{r}^{11} & \gamma_{r}^{21} & 0 \\
\gamma_{r}^{21} & \gamma_{r}^{22} & 0 \\
0 & 0 & \gamma_{r}^{33}
\end{array}\right) .
$$

Time derivative of (B.5) can be written as,

$$
\dot{\gamma}_{r}=\frac{\partial}{\partial t}\left(\begin{array}{ccc}
\gamma_{r}^{11} & \gamma_{r}^{21} & 0 \\
\gamma_{r}^{21} & \gamma_{r}^{22} & 0 \\
0 & 0 & \gamma_{r}^{33}
\end{array}\right)+\left(\begin{array}{ccc}
-2 C \gamma_{r}^{21} & -C \gamma_{r}^{22} & 0 \\
-C \gamma_{r}^{22} & 0 & 0 \\
0 & 0 & 0
\end{array}\right) .
$$

Finally, the three dimensional formulation of the equations (8) to (10) for the case of the vortex flow can be written as,

$$
\begin{aligned}
& \left(\begin{array}{ccc}
\tau^{11} & 0 & 0 \\
0 & \tau^{11} & 0 \\
0 & 0 & \tau^{33}
\end{array}\right)=G_{0} \lambda\left(\begin{array}{ccc}
\gamma_{r}^{11} & \gamma_{r}^{21} & 0 \\
\gamma_{r}^{21} & \gamma_{r}^{22} & 0 \\
0 & 0 & \gamma_{r}^{33}
\end{array}\right)+\mu\left(\begin{array}{ccc}
0 & -\frac{2 C}{r^{2}} & 0 \\
-\frac{2 C}{r^{2}} & 0 & 0 \\
0 & 0 & 0
\end{array}\right), \\
& \dot{\lambda}=\alpha(1-\lambda)-|\tau: \dot{\gamma}| \beta \lambda, \\
& \frac{\partial}{\partial t}\left(\begin{array}{ccc}
\gamma_{r}^{11} & \gamma_{r}^{21} & 0 \\
\gamma_{r}^{21} & \gamma_{r}^{22} & 0 \\
0 & 0 & \gamma_{r}^{33}
\end{array}\right)=\left(\begin{array}{ccc}
2 C \gamma_{r}^{21} & C \gamma_{r}^{22} & 0 \\
C \gamma_{r}^{22} & 0 & 0 \\
0 & 0 & 0
\end{array}\right)+\left(\begin{array}{ccc}
0 & -\frac{2 C}{r^{2}} & 0 \\
-\frac{2 C}{r^{2}} & 0 & 0 \\
0 & 0 & 0
\end{array}\right) \\
& -\frac{(1-\lambda) \alpha}{\lambda}\left(\begin{array}{ccc}
\gamma_{r}^{11} & \gamma_{r}^{21} & 0 \\
\gamma_{r}^{21} & \gamma_{r}^{22} & 0 \\
0 & 0 & \gamma_{r}^{33}
\end{array}\right)
\end{aligned}
$$

\section{References}

[1] M. L. Baker, J. H. Baas, J. Malarkey, R. Silva Jacinto, M. J. Craig, I. A. Kane, S. Barker, The Effect of Clay Type On The Properties of Cohesive Sediment Gravity Flows And Their Deposits, Jornal of Sedimentary Research 87 (2017) 1176-1195. doi:10.2110/jsr.2017.63.

[2] T. Healy, Y. Wang, J. A. Healy, Muddy Coasts of the World: Processes, Deposits and Function, Proceedings in Marine Science, Elsevier Science, 2002.

[3] J. C. Winterwerp, W. G. M. van Kesteren, Introduction to the Physics of Cohesive Sediment in the Marine Environment, Elsevier, 2004.

[4] D. S. Van Maren, J. C. Winterwerp, Z. Y. Wang, Q. Pu, Suspended sediment dynamics and morphodynamics in the Yellow River, China, Sedimentology 56 (3) (2009) 785-806. doi:10.1111/j.1365-3091.2008.00997.x. 
[5] P. Coussot, N. Roussel, S. Jarny, H. Chanson, Continuous or catastrophic solid-liquid transition in jammed systems, Physics of Fluids 17 (1). doi:10.1063/1.1823531.

[6] J. H. Baas, J. L. Best, J. Peakall, Predicting bedforms and primary current stratification in cohesive mixtures of mud and sand, Journal of the Geological Society 173 (1) (2016) 12-45. doi:10.1144/jgs2015-024.

[7] P. Coussot, Q. D. Nguyen, H. T. Huynh, D. Bonn, Avalanche behavior in yield stress fluids, Physical Review Letters 88 (17) (2002) 1755011-1755014. doi:10.1103/PhysRevLett.88.175501.

[8] P. Coussot, Q. D. Nguyen, H. T. Huynh, D. Bonn, Viscosity bifurcation in thixotropic, yielding fluids, Journal of Rheology 46 (3) (2002) 573-589. doi:10.1122/1.1459447.

[9] F. Pignon, A. Magnin, J.-M. Piau, Thixotropic colloidal suspensions and flow curves with minimum: Identification of flow regimes and rheometric consequences, Journal of Rheology 40 (1996) 573-587. doi:10.1122/1.550759.

[10] N. Hermidas, J. T. Eggenhuisen, R. Silva Jacinto, S. M. Luthi, F. Toth, F. Pohl, A Classification of Clay-Rich Subaqueous Density Flow Structures, Journal of Geophysical Research 123 (5) (2018) 945-966. doi:10.1002/2017JF004386.

[11] A. S. Michaels, J. C. Bolger, Settling rates and sediment volumes of flocculated kaolin suspensions, Industrial and Engineering Chemistry Fundamentals 1 (1) (1962) 24-33. doi:10.1021/i160001a004.

[12] J. Billingham, J. W. J. Ferguson, Laminar, unidirectional flow of a thixotropic fluid in a circular pipe, Journal of Non-Newtonian Fluid Mechanics 47 (1993) 21-55. doi:10.1016/0377-0257(93)80043-B

[13] C. F. Goodeve, A General Theory of Thixotropy and Viscosity, Trans. Faraday Soc. 35 (1939) 342-358. doi:10.1039/TF9393500342.

[14] F. Moore, The rheology of ceramic slips and bodies, Transactions of the British Ceramic Society 58 (1959) 470494.

[15] S. J. Hahn, T. Ree, H. Eyring, Flow Mechanism of Thixotropic Substances, Industrial and Engineering Chemistry 51 (7) (1959) 856-857. doi:10.1021/ie50595a038.

[16] S. Peter, Zur Theorie der Rheopexie, Rheologica Acta 3 (1964) 178-180. doi:10.1007/BF01668885.

[17] R. S. Rivlin, The hydrodynamics of non-Newtonian fluids. I, Proceedings of the Royal Society 193 (1033) (1948) 260-281. doi:10.1098/rspa.1948.0044.

[18] R. S. Rivlin, The hydrodynamics of non-Newtonian fluids . II, proceedings of cambridge philosophical society 45 (1) (1949) 88-91. doi:10.1017/S0305004100000463.

[19] D. C.-H. Cheng, F. Evans, Phenomenological characterization of the rheological behaviour of inelastic reversible thixotropic and antithixotropic fluids, British Journal of Applied Physics 16 (1965) 1599-1617. doi:10.1088/0508$3443 / 16 / 11 / 301$

[20] D. Hewitt, N. Balmforth, Thixotropic gravity currents, Journal of Fluid Mechanics 727 (2013) 56-82. doi:10.1017/jfm.2013.235.

[21] E. a. Toorman, Modelling the thixotropic behaviour of dense cohesive sediment suspensions, Rheologica Acta 36 (1997) 56-65. doi:10.1007/BF00366724.

[22] C. J. Dimitriou, G. H. McKinley, A comprehensive constitutive law for waxy crude oil: A thixotropic yield stress fluid, Soft Matter 10 (35) (2014) 6619-6644. doi:10.1039/c4sm00578c

[23] D. Acierno, F. La Mantia, G. Marrucci, G. Titomanlio, A non-linear viscoelastic model with structure-dependent relaxation times, Journal of Non-Newtonian Fluid Mechanics 1 (1976) 125-146. doi:10.1016/0377-0257(76)80012-

[24] P. Coussot, A. I. Leonov, J. M. Piau, Rheology of concentrated dispersed systems in a low molecular weight matrix, Journal of Non-Newtonian Fluid Mechanics 46 (1993) 179-211. doi:10.1016/0377-0257(93)85046-D.

[25] D. De Kee, C. F. Chan Man Fong, Rheological properties of structured fluids, Polymer Engineering \& Science 34 (5) (1994) 438-445. doi:10.1002/pen.760340510.

[26] C. F. C.M. Fong, G. Turcotte, D. De Kee, Modelling Steady and Transient Rheological Properties, Journal of Food Engineering 27 (1996) 63-70. doi:10.1016/0260-8774(94)00077-M.

[27] F. Yziquel, P. J. Carreau, M. Moan, P. A. Tanguy, Rheological modeling of concentrated colloidal suspensions, Journal of Non-Newtonian Fluid Mechanics 86 (1999) 133-155. doi:10.1016/S0377-0257(98)00206-7.

[28] P. R. de Souza Mendes, Modeling the thixotropic behavior of structured fluids, Journal of Non-Newtonian Fluid Mechanics 164 (1-3) (2009) 66-75. doi:10.1016/j.jnnfm.2009.08.005.

[29] P. Sollich, P. Hébraud, M. E. Cates, Rheology of Soft Glassy Materials, Physical Review Letters 78 (10). doi:10.1103/PhysRevLett.78.2020.

[30] S. M. Fielding, P. Sollich, M. E. Cates, Ageing and Rheology in Soft Materials, Journal of Rheology 44 (2). doi: $10.1122 / 1.551088$.

[31] M. L. Falk, J. S. Langer, Dynamics of viscoplastic deformation in amorphous solids, Physical Review E 57 (6) (1998) 7192-7205. doi:10.1103/PhysRevE.57.7192.

[32] L. Bocquet, A. Colin, A. Ajdari, Kinetic theory of plastic flow in soft glassy materials, Physical Review Letters 103 (3) (2009) 1-4. doi:10.1103/PhysRevLett.103.036001.

[33] A. Mujumdar, A. N. Beris, A. B. Metzner, Transient phenomena in thixotropic systems, Journal of Non-Newtonian 
Fluid Mechanics 102 (2002) 157-178. doi:10.1016/s0377-0257(01)00176-8

[34] K. Dullaert, J. Mewis, A structural kinetics model for thixotropy, Journal of Non-Newtonian Fluid Mechanics 139 (2006) 21-30. doi:10.1016/j.jnnfm.2006.06.002.

[35] P. Coussot, H. Tabuteau, X. Chateau, L. Tocquer, G. Ovarlez, Aging and solid or liquid behavior in pastes, Journal of Rheology 50 (6) (2006) 975-994. doi:10.1122/1.2337259.

[36] C. Derec, G. Ducouret, A. Ajdari, F. Lequeux, Aging and nonlinear rheology in suspensions of polyethylene oxideprotected silica particles, Physical Review E 67 (6) (2003) 9. doi:10.1103/PhysRevE.67.061403.

[37] H. V. Olphen, An introduction to clay colloid chemistry: for clay technologists, geologists, and soil scientists, Wiley, 1977.

[38] P. Coussot, Mudflow Rheology and Dynamics, IAHR Monographs, Taylor \& Francis, 1997.

[39] R. Roscoe, Mechanical Models for the Representation of Visco-Elastic Properties, British Journal of Applied Physics 1 (7) (1950) 171-173. doi:10.1088/0508-3443/1/7/302.

[40] R. Mas, A. Magnin, Rheology of colloidal suspensions: Case of lubricating greases, Journal of Rheology 38 (4) (1994) 889-908. doi:10.1122/1.550598.

[41] R. B. Bird, R. C. Armstrong, O. Hassager, Dynamics of Polymeric Liquids, 2nd Edition, John Wiley \& Sons, 1987.

[42] G. C. Holmes, The Use of Hyperbolic Cosines in Solving Cubic Polynomials, The Mathematical Gazette 86 (507) (2002) 473-477. doi:10.2307/3621149.

[43] A. S. Yoshimura, R. K. Prud'homme, Response of an elastic Bingham fluid to oscillatory shear, Rheologica Acta 26 (5) (1987) 428-436. doi:10.1007/BF01333843.

[44] L. F. Shampine, M. W. Reichelt, The MATLAB ODE Suite, SIAM Journal on Scientific Computing 18 (1) (1997) 1-22. doi:10.1137/S1064827594276424.

[45] R. I. Tanner, Engineering Rheology, Oxford Engineering Science Series, OUP Oxford, 2000. 